\title{
Filtering and Fault Detection for Nonlinear Systems with Polynomial Approximation
}

\author{
Yang Liu ${ }^{\mathrm{a}}$, Zidong Wang ${ }^{\mathrm{b}}$, Xiao He ${ }^{\mathrm{a}}$, D. H. Zhou ${ }^{\mathrm{a}, *}$ \\ ${ }^{a}$ Department of Automation, TNList, Tsinghua University, Beijing 100084, P. R. China. \\ ${ }^{\mathrm{b}}$ Department of Computer Science, Brunel University London, Uxbridge, Middlesex, UB8 3PH, United Kingdom.
}

\begin{abstract}
This paper is concerned with polynomial filtering and fault detection problems for a class of nonlinear systems subject to additive noises and faults. The nonlinear functions are approximated with polynomials of a chosen degree. Different from the traditional methods, the approximation errors are not discarded but formulated as low-order polynomial terms with normbounded coefficients. The aim of the filtering problem is to design a least squares filter for the formulated nonlinear system with uncertain polynomials, and an upper bound of the filtering error covariance is found and subsequently minimized at each time step. The desired filter gain is obtained by recursively solving a set of Riccati-like matrix equations, and the filter design algorithm is therefore applicable for online computation. Based on the established filter design scheme, the fault detection problem is further investigated where the main focus is on the determination of the threshold on the residual. Due to the nonlinear and time-varying nature of the system under consideration, a novel threshold is determined that accounts for the noise intensity and the approximation errors, and sufficient conditions are established to guarantee the fault detectability for the proposed fault detection scheme. Comparative simulations are exploited to illustrate that the proposed filtering strategy achieves better estimation accuracy than the conventional polynomial extended Kalman filtering approach. The effectiveness of the associated fault detection scheme is also demonstrated.
\end{abstract}

Key words: Polynomial filtering; nonlinear systems; polynomial approximation; fault detection; adaptive threshold; recursive algorithm, Kronecker power.

\section{Introduction}

The past few decades have seen the nonlinear state estimation problem as a recurring research theme due to the pervasive existence of nonlinearities in almost all real-world industrial systems. If not adequately dealt with, the intrinsic nonlinearities may lead to undesirable dynamic behaviors such as oscillation or even instability. Indeed, the nonlinear analysis issue has been the main stream of research for systems control and estimation problems attracting researchers from a variety of disciplines. So far, much effort has been devoted to the estimation/filtering problems for nonlinear systems, see e.g. $[2,4,7,11-13,19,21,23,27,35,36]$ and the references therein. Among others, the renowned extended Kalman filter (EKF) algorithm has proved to be an effective method to solve the estimation problem for nonlinear systems in the least mean square sense. Recently, considerable attention has been paid to the performance

\footnotetext{
* Corresponding author.

Email address: zdh@mail.tsinghua.edu.cn (D. H. Zhou).
}

improvement of the traditional EKF with respect to the insensitivity to the parameter uncertainties as well as the capability of handling nonlinearities [22,32,38]. When the system states and observations are polynomial with additive Gaussian white noises, the mean-square filter has been designed where the statistical characteristics of Gaussian distribution have been made use of to recursively calculate the filtering error covariance $[1,3,5]$.

Polynomial extended Kalman filter (PEKF) is an extension of EKF with aim to cater for inherent nonlinearies using polynomial approximations. Traditional EKF is only concerned with the linear term and simply ignores the linearization error, while PEKF considers the Carleman approximation of a nonlinear system of a given order $\mu[26]$. The order could be determined according to the form of the nonlinearity and the estimation performance specification. In this sense, the PEKF is more applicable than EKF as far as the accuracy is concerned. When the order $\mu=1$, PEKF reduces to conventional EKF. A PEKF is designed to cope with an augmented state which is made of Kronecker powers of the original 
state [15]. Due to its higher accuracy than that of EKF, the PEKF has stirred quite a lot research attention and many corresponding results have been reported in the literature $[14,16,17,28]$. Nevertheless, the PEKF approach still ignores the Carleman approximation errors which would give rise to certain conservatism especially when the nonlinearities are severe.

In theory, well-behaved nonlinear functions could only be approximated accurately by polynomials whose orders approach infinity. In engineering systems, however, the polynomials with extremely high orders are difficult to be realized. A feasible way is to determine the finite order of the polynomials for satisfactory approximation of the nonlinear dynamics according to the degree of the nonlinearities and the nature of the research problem. As such, the unavoidable high-order approximation errors would be impacting on the estimation performances that should be taken into account. While EKF and PEKF work quite well for system with relatively low degree of nonlinearities, the classical EKF algorithm ignores the linearization errors and most available PEKF approaches discard the Carleman approximation errors. It is noted that the approximation errors differ greatly from each other due to various forms of nonlinearities. Instead of being simply dropped, the approximation errors do offer further room for improving the estimation accuracy if properly coped with. This seemingly natural idea, unfortunately, would inevitably bring us substantial challenges when calculating the covariances of the estimation errors in the least mean square sense since the approximation errors could not be exactly known. Moreover, coupled with both the low-order terms and external disturbances, the approximate errors would become very complicated to analyze. These identified difficulties motivate us to address the PEKF problem by allowing for the Carleman approximation error with aim to obtain higher approximation accuracy and better estimation performance.

The fault detection (FD) problem is another active research topic in control engineering due primarily to the increasingly higher and higher safety requirements. Yet, the FD problem provides an ideal platform to demonstrate the applicability of the polynomial filter technique to be developed. Since a properly designed filter could generate residual signal so as to efficiently detect abnormal changes in the system, filter/observer based FD has become a common technique. In filter/observer based FD methods, a fault would be detected and diagnosed effectively via comparing the actual system output with the filter output signal since the faults would normally bring in unexpected variations in the system measurement. To date, a great number of results have been published on this issue, see $[18,33,41,44]$ and the references therein. Residual evaluation, which consists of threshold and decision logic, is critical to the performance of FD. For systems varying with time or parameters, the adaptive threshold has been of particular research importance for its better trackability and faster self-adjustment as compared with the constant threshold.

So far, many existing results have focused on adaptive threshold generation for linear systems [29-31,43]. However, the corresponding results for nonlinear systems have been scattered in spite of their engineering significance $[24,34]$. In $[40,42]$, the nonlinear dynamics have been assumed to be uniformly bounded and the bounds have been utilized to determine the adaptive threshold. In the context of polynomial fault detection for nonlinear systems, the adaptive threshold determination problem is essentially difficult mainly for two reasons: 1) the expressions of the disturbances and approximation errors are sophisticated and their influences on the threshold remain unclear; and 2) it is non-trivial to calculate the bounds of the disturbances and approximation errors in order to propose a reasonable fault detection threshold. Up to date, the polynomial fault detection scheme has not been fully investigated, not to mention the case where the Carleman approximation errors are taken into consideration. This constitutes another motivation of our present work.

Summarizing the discussions made above, in this paper, both the polynomial filtering and fault detection problems are thoroughly investigated for a class of nonlinear systems. The Carleman approximation of a given order is introduced to approximate the nonlinear functions. In contrast to existing literature, the high-order approximation errors are explicitly taken into account in terms of low-order polynomials with uncertain but bounded coefficients. A time-varying filter is first designed at each time step to guarantee that the filtering error covariance is bounded in the fault-free case. Such a bound is subsequently minimized with respect to a properly designed filter gain. To show the applicability of the proposed filter scheme, a fault detection strategy is then proposed consisting of the calculation of adaptive threshold and decision logic. The filter gain and adaptive threshold are determined using the information from the approximation errors and additive disturbances. With the fault detection strategy, the fault detectability is also investigated.

The main contribution of the paper can be highlighted as follows: 1) a polynomial estimation scheme for a class of nonlinear systems is presented by taking account of the Carleman approximation errors, which leads to higher estimation accuracy; 2) an upper bound of the estimation error covariance in the polynomial scheme is calculated and minimized by designing an appropriate filter gain; 3) adaptive fault detection threshold is developed for the desired polynomial filter to realize efficient detection and the fault detectability is analyzed; and 4) the proposed algorithms for both the filter gain design and the adaptive threshold computation are recursive and therefore suitable for online applications. The rest of paper is organized as follows. In Section 2, the Carleman ap- 
proximation and the approximation error analysis are introduced for nonlinear systems. The polynomial filter design problem is solved in Section 3 and the adaptive fault detection scheme is established in Section 4. A simulation example is illustrated in Section 5 and the paper is concluded in Section 6.

Notations. The notation used in the paper is standard. $\mathbb{R}^{n}$ and $\mathbb{R}^{n \times m}$ denote, respectively, the $n$-dimensional Euclidean space and the set of all $n \times m$ real matrices. The superscripts $A^{T}$ and $A^{-1}$ denote the transpose and inverse of matrix $A$, respectively. The notation $X \geq Y$ (respectively, $X>Y$ ), where $\mathrm{X}$ and $\mathrm{Y}$ are symmetric matrices, means that $X-Y$ is positive semidefinite (respectively, positive definite). $I$ is the identity matrix with compatible dimension. $\mathbb{E}\{x\}$ stands for the expectation of the stochastic variable $x$. $\|A\|$ denotes the spectral norm of matrix $A$, and $\|x\|$ refers to the Euclidean norm of vector $x . \otimes$ is the Kronecker product defined as $A \otimes B=\left[\begin{array}{ccc}a_{1,1} B & \cdots & a_{1, n} B \\ \vdots & \ddots & \vdots \\ a_{m, 1} B & \cdots & a_{m, n} B\end{array}\right] . x^{[m]}$ represents the $m$ th

Kronecker power of vector $x$, where $x^{[m]}=x^{[m-1]} \otimes x$ and $x^{[0]}=1[6,10]$.

\section{Problem Formulation}

Consider the following class of stochastic discrete-time nonlinear systems:

$$
\left\{\begin{aligned}
x_{k+1} & =g\left(x_{k}, u_{k}\right)+v_{k}+f_{k} \\
y_{k} & =h\left(x_{k}\right)+w_{k}
\end{aligned}\right.
$$

where $x_{k} \in \mathbb{R}^{n}$ is the system state; $y_{k} \in \mathbb{R}^{b}$ is the measurement output; $u_{k} \in \mathbb{R}^{l}$ is the control input; $f_{k} \in \mathbb{R}^{n}$ is the additive fault; $g: \mathbb{R}^{n} \times \mathbb{R}^{l} \mapsto \mathbb{R}^{n}$ and $h: \mathbb{R}^{n} \mapsto \mathbb{R}^{b}$ are $\mu+1$ times continuously differentiable nonlinear maps. The state noise $v_{k} \in \mathbb{R}^{n}$ and the output noise $w_{k} \in \mathbb{R}^{b}$ are uncorrelated zero-mean sequences. The initial state $x_{0}$ is random and independent of the noises. It is assumed that

$$
\mathbb{E}\left\{x_{0}^{[i]}\right\}=\zeta_{0, i}, \quad \mathbb{E}\left\{v_{k}^{[i]}\right\}=\xi_{v, k, i}, \quad \mathbb{E}\left\{w_{k}^{[i]}\right\}=\xi_{w, k, i},
$$

for all $i=1,2, \ldots, 2 \mu$, where $\zeta_{0, i}, \xi_{v, k, i}$, and $\xi_{w, k, i}$ are known vectors. Meanwhile, $x_{0}^{[i]}, v_{k}^{[i]}$, and $w_{k}^{[i]}$ are assumed to be distributed in bounded domains and the following inequalities hold definitely:

$$
\left\|x_{0}^{[i]}\right\| \leq s_{0, i}, \quad\left\|v_{k}^{[i]}\right\| \leq s_{v, k, i}, \quad\left\|w_{k}^{[i]}\right\| \leq s_{w, k, i},
$$

where $s_{0, i}, s_{v, k, i}$ and $s_{w, k, i}$ are known scalars for all $i=$ $1,2, \ldots, \mu$. The fault $f_{k}$ is assumed to be norm-bounded so that the measurements and the estimated states will not go infinity in a finite time in the faulty case.

\subsection{Polynomial approximation of nonlinear functions}

Consider the sequences $x_{k+1}^{[m]}$ and $y_{k}^{[m]}$, the Kronecker powers of the state and measurement around the state estimate $\tilde{x}_{k}$ for $m=0,1, \ldots, \mu$. With Taylor polynomials around $\tilde{x}_{k}$, when the nonlinear functions are $\mu+1$ times continuously differentiable, we have

$$
\left\{\begin{aligned}
x_{k+1}^{[m]}= & \sum_{i=0}^{\mu} G_{m, i}\left(\tilde{x}_{k}, u_{k}, v_{k}, f_{k}\right)\left(x_{k}-\tilde{x}_{k}\right)^{[i]} \\
& +G_{m, \mu+1}\left(x_{k}, \tilde{x}_{k}, u_{k}, v_{k}, f_{k}\right)\left(x_{k}-\tilde{x}_{k}\right)^{[\mu+1]} \\
y_{k}^{[m]}= & \sum_{i=0}^{\mu} H_{m, i}\left(\tilde{x}_{k}, w_{k}\right)\left(x_{k}-\tilde{x}_{k}\right)^{[i]} \\
& +H_{m, \mu+1}\left(x_{k}, \tilde{x}_{k}, w_{k}\right)\left(x_{k}-\tilde{x}_{k}\right)^{[\mu+1]}
\end{aligned}\right.
$$

where $\tilde{x}_{\theta_{k}}=\Theta_{k} x_{k}+\left(I-\Theta_{k}\right) \tilde{x}_{k}, \Theta_{k}=\operatorname{diag}\left\{\theta_{1 k}, \ldots, \theta_{n k}\right\}$, $\theta_{i k} \in[0,1]$ for all $i=1,2, \ldots, n$, and

$$
\begin{aligned}
& G_{m, i}\left(\tilde{x}_{k}, u_{k}, v_{k}, f_{k}\right) \\
= & \left.\frac{1}{i !}\left(\nabla_{x}^{[i]} \otimes(g+v+f)^{[m]}\right)\right|_{\left(x=\tilde{x}_{k}, u=u_{k}, v=v_{k}, f=f_{k}\right)}, \\
& H_{m, i}\left(\tilde{x}_{k}, w_{k}\right) \\
= & \left.\frac{1}{i !}\left(\nabla_{x}^{[i]} \otimes(h+w)^{[m]}\right)\right|_{\left(x=\tilde{x}_{k}, w=w_{k}\right)},
\end{aligned}
$$

where the operation $\nabla_{x}^{[i]} \otimes$ applied to a function $\chi(x)$ is defined as:

$$
\begin{aligned}
\nabla_{x}^{[0]} \otimes \chi & =\chi, \\
\nabla_{x}^{[i+1]} \otimes \chi & =\nabla_{x} \otimes\left(\nabla_{x}^{[i]} \otimes \chi\right), i>0,
\end{aligned}
$$

with $\nabla_{x}=\left[\partial / \partial x_{1}, \ldots, \partial / \partial x_{n}\right]$.

In order to obtain a least squares filter and determine the adaptive threshold for fault detection, now we consider system (1) with $f_{k}=0$. Firstly we focus on the expression of the remainder terms. Denote

$\nabla_{x}^{[\mu]} \otimes(g+v)^{[m]}=\left[\varepsilon_{1, \mu, m}(x, u, v), \ldots, \varepsilon_{n^{m}, \mu, m}(x, u, v)\right]^{T}$,

where $\varepsilon_{i, \mu, m}(x, u, v): \mathbb{R}^{n} \times \mathbb{R}^{l} \times \mathbb{R}^{n} \mapsto \mathbb{R}^{n^{\mu}}$ for all $i=$ $1, \ldots, n^{m}$. It can be easily verified that

$$
\begin{aligned}
& G_{m, \mu+1}\left(x_{k}, \tilde{x}_{k}, u_{k}, v_{k}, 0\right)\left(x_{k}-\tilde{x}_{k}\right)^{[\mu+1]} \\
= & \mathfrak{G}\left(x_{k}, \tilde{x}_{k}, u_{k}, v_{k}\right)\left(x_{k}-\tilde{x}_{k}\right)^{[\mu]},
\end{aligned}
$$


where

$$
\begin{aligned}
& \mathfrak{G}\left(x_{k}, \tilde{x}_{k}, u_{k}, v_{k}\right)=\frac{1}{(\mu+1) !} \\
& \times\left[\begin{array}{c}
\left(x_{k}-\tilde{x}_{k}\right)^{T}\left(\nabla_{x}^{T} \otimes \varepsilon_{1, \mu, m}^{T}\left(\tilde{x}_{\theta_{k}}, u_{k}, v_{k}\right)\right) \\
\vdots \\
\left(x_{k}-\tilde{x}_{k}\right)^{T}\left(\nabla_{x}^{T} \otimes \varepsilon_{n^{m}, \mu, m}^{T}\left(\tilde{x}_{\theta_{k}}, u_{k}, v_{k}\right)\right)
\end{array}\right] .
\end{aligned}
$$

We assume that $x_{k}$ is bounded in an ellipsoid of center $\tilde{x}_{k}$ and shape matrix $E_{k}$, i.e. $x_{k}=\tilde{x}_{k}+E_{k} z_{k}$, for some $z_{k} \in \mathbb{R}^{n}$ and $\left\|z_{k}\right\| \leq 1$. Since the nonlinear functions $\nabla_{x}^{T} \otimes \varepsilon_{i, \mu, m}^{T}\left(\tilde{x}_{\theta_{k}}, u_{k}, v_{k}\right)$ are assumed to be continuous, their norm reaches an extremum on the bounded ellipsoid domain, thus there exist constants $\bar{m}_{1, k}, \ldots, \bar{m}_{n^{m}, k}$, such that for $i=1,2, \ldots, n^{m}$,

$$
\left\|\nabla_{x}^{T} \otimes \varepsilon_{i, \mu, m}^{T}\left(\tilde{x}_{\theta_{k}}, u_{k}, v_{k}\right)\right\| \leq \bar{m}_{i, k} .
$$

With $x_{k}=\tilde{x}_{k}+E_{k} z_{k}$ and $\left\|z_{k}\right\| \leq 1$, it follows directly that

$$
\left\|\left(x_{k}-\tilde{x}_{k}\right)^{T}\left(\nabla_{x}^{T} \otimes \varepsilon_{i, \mu, m}^{T}\left(\tilde{x}_{\theta_{k}}, u_{k}, v_{k}\right)\right)\right\| \leq \bar{m}_{i, k}\left\|E_{k}\right\|,
$$

therefore there exist vectors $\varsigma_{i, k} \in \mathbb{R}^{n^{\mu}}$ such that $\left\|\varsigma_{i, k}\right\| \leq$ 1 and

$$
\left(x_{k}-\tilde{x}_{k}\right)^{T}\left(\nabla_{x}^{T} \otimes \varepsilon_{i, \mu, m}^{T}\left(\tilde{x}_{\theta_{k}}, u_{k}, v_{k}\right)\right)=\bar{m}_{i, k}\left\|E_{k}\right\| \varsigma_{i, k}^{T} .
$$

Denoting $\Xi_{k}=\left[\varsigma_{1, k}, \ldots, \varsigma_{n^{m}, k}\right]^{T}$, we have

$\mathfrak{G}\left(\tilde{x}_{\theta_{k}}, u_{k}, v_{k}\right)=\frac{1}{(\mu+1) !}\left\|E_{k}\right\| \operatorname{diag}\left\{\bar{m}_{1, k}, \ldots, \bar{m}_{n^{m}, k}\right\} \Xi_{k}$.

From the definition of $\Xi_{k}$ and $\left\|\varsigma_{i, k}\right\| \leq 1$, it is obvious that $\left\|\Xi_{k}\right\| \leq \sqrt{n^{m}}$. Therefore, denote

$$
L_{g, m, k}=\frac{\sqrt{n^{m}}}{(\mu+1) !}\left\|E_{k}\right\| \operatorname{diag}\left\{\bar{m}_{1, k}, \ldots, \bar{m}_{n^{m}, k}\right\}
$$

then $\mathfrak{G}\left(x_{k}, \tilde{x}_{k}, u_{k}, v_{k}\right)$ can be written as

$$
\mathfrak{G}\left(x_{k}, \tilde{x}_{k}, u_{k}, v_{k}\right)=L_{g, m, k} \Delta_{g, m, k},
$$

where $L_{g, m, k}$ and $\Delta_{g, m, k}$ are problem-dependent matrices with $\left\|\Delta_{g, m, k}\right\| \leq 1$. Similarly, we can have that

$$
\begin{aligned}
& H_{m, \mu+1}\left(x_{k}, \tilde{x}_{k}, w_{k}\right)\left(x_{k}-\tilde{x}_{k}\right)^{[\mu+1]} \\
= & L_{h, m, k} \Delta_{h, m, k}\left(x_{k}-\tilde{x}_{k}\right)^{[\mu]},
\end{aligned}
$$

where $L_{h, m, k}$ and $\Delta_{h, m, k}$ are problem-dependent matrices with $\left\|\Delta_{h, m, k}\right\| \leq 1$ as well.
Remark 1 The way of processing the high-order approximation errors is along the similar line of coping with the linearization errors in [8, 20, 39] where linear terms with norm-bounded matrix coefficients have been utilized to formulate the linearization errors. Different from [8, 20, 39], in this paper, polynomial terms of orders higher than $\mu$, namely, the approximation errors, are formulated as terms of lower orders with norm-bounded matrix coefficients. The unknown bounded matrices $\Delta_{g, m, k}$ and $\Delta_{h, m, k}$ are functions of both $x_{k}$ and $\tilde{x}_{k}$, and they are not expressed as $\Delta_{g, m, k}\left(x_{k}, \tilde{x}_{k}\right)$ and $\Delta_{h, m, k}\left(x_{k}, \tilde{x}_{k}\right)$ only for simplicity. Notice that the approximation errors have been ignored in previous PEKF studies, which may lead to unsatisfactory estimation performance especially when the encountered nonlinearities are severe. Obviously, the approximation errors have a great influence on the estimation accuracy, and the novel error formulation (18) helps to achieve a better approximation and estimation performance in the sequel, which will be demonstrated in the simulation.

\subsection{The polynomial nonlinear systems}

Before proceeding further, the following lemma is introduced.

Lemma 1 [9] For any integer $h \geq 0$ and for any $a, b \in$ $\mathbb{R}^{n}$, we have

$$
(a+b)^{[h]}=\sum_{k=0}^{h} M_{h}^{k}\left(a^{[k]} \otimes b^{[h-k]}\right)
$$

with a set of suitably defined matrices $M_{h}^{k} \in \mathbb{R}^{n^{h} \times n^{h}}(k=$ $0,1, \ldots, h)$.

With Lemma 1, (17), (18) and the fact that

$$
(A \otimes B)(C \otimes D)=(A C) \otimes(B D),
$$

(4) in the fault-free case can be written as follows:

$$
\left\{\begin{aligned}
x_{k+1}^{[m]}= & \sum_{j=0}^{\mu} A_{m, j, k} x_{k}^{[j]}+L_{g, m, k} \Delta_{g, m, k} \sum_{j=0}^{\mu} \Upsilon_{j, k} x_{k}^{[j]} \\
& +v_{m, k}, \\
y_{k}^{[m]}= & \sum_{j=0}^{\mu} C_{m, j, k} x_{k}^{[j]}+L_{h, m, k} \Delta_{h, m, k} \sum_{j=0}^{\mu} \Upsilon_{j, k} x_{k}^{[j]} \\
& +w_{m, k},
\end{aligned}\right.
$$

where

$$
\begin{aligned}
A_{m, j, k}= & \sum_{i=j}^{\mu} \sum_{p=0}^{m} \frac{1}{i !} M_{m}^{p}\left(\left(\nabla_{x}^{[i]} \otimes g^{[p]}\right) \otimes \xi_{v, k, m-p}\right) \\
& \times M_{i}^{j}\left(I_{n^{j}} \otimes\left(-\tilde{x}_{k}\right)^{[i-j]}\right),
\end{aligned}
$$




$$
\begin{aligned}
C_{m, j, k}= & \sum_{i=j}^{\mu} \sum_{p=0}^{m} \frac{1}{i !} M_{m}^{p}\left(\left(\nabla_{x}^{[i]} \otimes h^{[p]}\right) \otimes \xi_{w, k, m-p}\right) \\
& \times M_{i}^{j}\left(I_{n^{j}} \otimes\left(-\tilde{x}_{k}\right)^{[i-j]}\right), \\
v_{m, k}= & \sum_{i=0}^{\mu} \sum_{p=0}^{m} \frac{1}{i !} M_{m}^{p}\left(\left(\left(\nabla_{x}^{[i]} \otimes g^{[p]}\right)\left(x_{k}-\tilde{x}_{k}\right)^{[i]}\right)\right. \\
& \left.\otimes\left(v_{k}^{[m-p]}-\xi_{v, k, m-p}\right)\right), \\
w_{m, k}= & \sum_{i=0}^{\mu} \sum_{p=0}^{m} \frac{1}{i !} M_{m}^{p}\left(\left(\left(\nabla_{x}^{[i]} \otimes h^{[p]}\right)\left(x_{k}-\tilde{x}_{k}\right)^{[i]}\right)\right. \\
& \left.\otimes\left(w_{k}^{[m-p]}-\xi_{w, k, m-p}\right)\right), \\
\Upsilon_{j, k}= & M_{\mu}^{j}\left(I_{n^{j}} \otimes\left(-\tilde{x}_{k}\right)^{[\mu-j]}\right) .
\end{aligned}
$$

Denote the following augmented state and measurement:

$$
x_{k}^{(\mu)}=\left[\begin{array}{c}
1 \\
x_{k} \\
x_{k}^{[2]} \\
\vdots \\
x_{k}^{[\mu]}
\end{array}\right], y_{k}^{(\mu)}=\left[\begin{array}{c}
1 \\
y_{k} \\
y_{k}^{[2]} \\
\vdots \\
y_{k}^{[\mu]}
\end{array}\right],
$$

then (21) can be written as:

$$
\left\{\begin{array}{c}
x_{k+1}^{(\mu)}=A_{k} x_{k}^{(\mu)}+L_{g, k} \Delta_{g, k} \Upsilon_{k} x_{k}^{(\mu)}+v_{k}^{(\mu)} \\
y_{k}^{(\mu)}=C_{k} x_{k}^{(\mu)}+L_{h, k} \Delta_{h, k} \Upsilon_{k} x_{k}^{(\mu)}+w_{k}^{(\mu)}
\end{array}\right.
$$

where

$$
\begin{aligned}
& A_{k}=\left[\begin{array}{ccc}
A_{0,0, k} & \ldots & A_{0, \mu, k} \\
A_{1,0, k} & \ldots & A_{1, \mu, k} \\
\vdots & \ddots & \vdots \\
A_{\mu, 0, k} & \ldots & A_{\mu, \mu, k}
\end{array}\right], \\
& C_{k}=\left[\begin{array}{ccc}
C_{0,0, k} & \ldots & C_{0, \mu, k} \\
C_{1,0, k} & \ldots & C_{1, \mu, k} \\
\vdots & \ddots & \vdots \\
C_{\mu, 0, k} & \ldots & C_{\mu, \mu, k}
\end{array}\right], \\
& v_{k}^{(\mu)}=\left(v_{0, k}^{T}, \ldots, v_{\mu, k}^{T}\right)^{T}, w_{k}^{(\mu)}=\left(w_{0, k}^{T}, \ldots, w_{\mu, k}^{T}\right)^{T}, \\
& \Upsilon_{k}=\left[\Upsilon_{0, k}, \ldots, \Upsilon_{\mu, k}\right], L_{g, k}=\operatorname{diag}\left\{L_{g, 0, k}, \ldots, L_{g, \mu, k}\right\}, \\
& L_{h, k}=\operatorname{diag}\left\{L_{h, 0, k}, \ldots, L_{h, \mu, k}\right\}, \\
& \Delta_{g, k}=\operatorname{diag}\left\{\Delta_{g, 0, k}, \ldots, \Delta_{g, \mu, k}\right\}, \\
& \Delta_{h, k}=\operatorname{diag}\left\{\Delta_{h, 0, k}, \ldots, \Delta_{h, \mu, k}\right\},
\end{aligned}
$$

and it is straightforward to see that $\left\|\Delta_{g, k}\right\| \leq 1$ and $\left\|\Delta_{h, k}\right\| \leq 1$. It is noted that when $g\left(x_{k}, u_{k}\right)$ (respec- tively, $h\left(x_{k}\right)$ ) is linear, the parameter $L_{g, k}$ (respectively, $\left.L_{h, k}\right)$ is zero. From the facts that $\mathbb{E}\left\{v_{k}^{[i]}\right\}=\xi_{v, k, i}$ and $\mathbb{E}\left\{w_{k}^{[i]}\right\}=\xi_{w, k, i}$, it follows that $v_{k}^{(\mu)}$ and $w_{k}^{(\mu)}$ are both zero-mean. However, it is quite difficult to calculate $\mathbb{E}\left\{v_{k}^{(\mu)}\left(v_{k}^{(\mu)}\right)^{T}\right\}$ and $\mathbb{E}\left\{w_{k}^{(\mu)}\left(w_{k}^{(\mu)}\right)^{T}\right\}$ due to that $v_{k}^{(\mu)}$ and $w_{k}^{(\mu)}$ involve the system state, the state estimate, and the nonlinear dynamics.

\subsection{The filter and the fault detection problems}

For system (28), the filter to be designed is of the following form:

$$
\tilde{x}_{k+1}^{(\mu)}=A_{k} \tilde{x}_{k}^{(\mu)}+K_{k}\left(y_{k}^{(\mu)}-C_{k} \tilde{x}_{k}^{(\mu)}\right),
$$

where $\tilde{x}_{k}^{(\mu)}$ is the estimate of $x_{k}^{(\mu)}$ at time step $k$ with $\tilde{x}_{0}^{(\mu)}=\mathbb{E}\left\{x_{0}^{(\mu)}\right\} \cdot K_{k}$ is the filter gain to be determined.

We are now in a position to state the addressed polynomial filter and fault detection problems as follows. 1) We are interested in designing the filter gain $K_{k}$ in (30) for the system (28) such that the resulting estimation error covariance is bounded and such a bound is subsequently minimized. 2) Based on the proposed filter design scheme, the associated fault detection problem is to generate a residual signal whose threshold is adaptively computed by reflecting the approximation errors and external disturbances.

Remark 2 Based on the degrees of the nonlinearities and the engineering requirement, the integer $\mu$ could be chosen in advance. A bigger $\mu$ would lead to higher approximation accuracy at the cost of heavier computation burden. In this sense, the determination of $\mu$ should be made according to the trade-off between the estimation accuracy and the computation expense. Therefore, the polynomial filter to be developed is applicable in many nonlinear cases since the order could be selected adaptively. When $\mu=1$ and the approximation errors are not considered, the filter to be designed reduces to the conventional EKF.

Remark 3 Though the polynomial approximation errors could be represented in a mathematical way, they remain indeterminate to the designers because of the unknown matrix coefficients. As a result, the accurate estimation error covariance could not be obtained. A natural alternative is to find an upper bound of the estimation error covariance and then minimize it by a properly designed filter gain $K_{k}$. This way, the feasibility of the algorithm is enhanced and the robustness with respect to the approximation errors is maintained. After the determination of $K_{k}$, an adaptive fault detection strategy would be established accordingly. 
Remark 4 It is observed from (21)-(28) that, in the proposed filter (30), $A_{k}$ and $C_{k}$ are quite complicated since they are nonlinear functions of the estimated states, addressed nonlinear dynamics as well as the statistics of disturbances. Rather than $A\left(\tilde{x}_{k}^{(\mu)}\right)$ and $C\left(\tilde{x}_{k}^{(\mu)}\right)$, they are denoted by $A_{k}$ and $C_{k}$ for simplicity only. By no means are they similar to the known system matrices in the linear case. In the estimation process, these two parameters have to be updated at each time instant by fairly complicated computation.

\section{Polynomial Filter Design}

The following lemmas are essential in establishing the main results.

Lemma 2 [37] Given matrices $A, H, E$ and $F$ with appropriate dimensions such that $F F^{T} \leq I$. Let $X$ be a symmetric positive definite matrix and $\gamma$ be an arbitrary positive constant such that $\gamma^{-1} I>E X E^{T}$. Then the following inequality holds

$$
\begin{aligned}
(A+H F E) X(A+H F E)^{T} \leq & A\left(X^{-1}-\gamma E^{T} E\right)^{-1} A^{T} \\
& +\gamma^{-1} H H^{T} .
\end{aligned}
$$

Lemma 3 [20] For any two vectors $x, y \in \mathbb{R}^{n}$, the following inequality holds

$$
x y^{T}+y x^{T} \leq \varepsilon x x^{T}+\varepsilon^{-1} y y^{T}
$$

where $\varepsilon>0$ is a scalar.

Denote the estimation error as

$$
e_{k}=x_{k}^{(\mu)}-\tilde{x}_{k}^{(\mu)},
$$

and the estimation error covariance conditional on the observations $y_{j}(j=0, \ldots, k)$ as

$$
P_{k+1}=\mathbb{E}\left\{e_{k+1} e_{k+1}^{T} \mid y_{0}, \ldots, y_{k}\right\}
$$

For presentation convenience, $\tilde{x}_{k}^{(\mu)}, e_{k}$ and $P_{k}$ are partitioned as follows:

$$
\tilde{x}_{k}^{(\mu)}=\left[\begin{array}{c}
\tilde{x}_{0, k}^{(\mu)} \\
\tilde{x}_{1, k}^{(\mu)} \\
\vdots \\
\tilde{x}_{\mu, k}^{(\mu)}
\end{array}\right], e_{k}=\left[\begin{array}{c}
e_{0, k} \\
e_{1, k} \\
\vdots \\
e_{\mu, k}
\end{array}\right]
$$

$$
P_{k}=\left[\begin{array}{cccc}
P_{0,0, k} & P_{0,1, k} & \cdots & P_{0, \mu, k} \\
P_{1,0, k} & P_{1,1, k} & \cdots & P_{1, \mu, k} \\
\vdots & \vdots & \ddots & \vdots \\
P_{\mu, 0, k} & P_{\mu, 1, k} & \cdots & P_{\mu, \mu, k}
\end{array}\right]
$$

where $e_{i, k}, \tilde{x}_{i, k}^{(\mu)} \in \mathbb{R}^{n^{i}}$ and $P_{i, j, k} \in \mathbb{R}^{n^{i} \times n^{j}}$ for any $i, j=$ $0,1, \ldots, \mu$. It can be seen that $P_{i, j, 0}=\mathrm{st}_{n^{i}, n^{j}}^{-1}\left(\zeta_{0, i+j}\right)-$ $\zeta_{0, i} \zeta_{0, j}^{T}$, where $\mathrm{st}_{a, b}^{-1}$ is the inverse of the stack operator defined as: for a vector $\rho=\left[\rho_{1}, \ldots, \rho_{a \times b}\right], \operatorname{st}_{a, b}^{-1}(\rho)=$ $\left[\varrho_{i j}\right]_{a \times b}$, where $\varrho_{i j}=\rho_{(j-1) a+i}$.

The first goal of this paper is to design a filter in the form of (30) for system (28) such that an upper bound of the covariance of the filtering error $P_{k}$ can be provided and minimized. For this purpose, let us now deal with the covariance of the filtering error in the following lemma.

\section{Lemma 4 Denoting}

$$
\begin{aligned}
\check{A}_{k}= & \left(A_{k}-K_{k} C_{k}\right)+\sqrt{2}\left[L_{g, k},-K_{k} L_{h, k}\right] \\
& \times\left[\frac{1}{\sqrt{2}} \Delta_{g, k}^{T}, \frac{1}{\sqrt{2}} \Delta_{h, k}^{T}\right]^{T} \Upsilon_{k}, \\
\Psi_{k}^{v}= & \mathbb{E}\left\{v_{k}^{(\mu)}\left(v_{k}^{(\mu)}\right)^{T}\right\} \\
\Psi_{k}^{w}= & \mathbb{E}\left\{w_{k}^{(\mu)}\left(w_{k}^{(\mu)}\right)^{T}\right\}
\end{aligned}
$$

the covariance of the filtering error in (34) obeys the following recursion:

$$
\begin{aligned}
P_{k+1}= & \mathbb{E}\left\{\check{A}_{k} P_{k} \check{A}_{k}^{T}\right\}+2\left[L_{g, k},-K_{k} L_{h, k}\right] \\
& \times \mathbb{E}\left\{\left[\frac{1}{\sqrt{2}} \Delta_{g, k}^{T}, \frac{1}{\sqrt{2}} \Delta_{h, k}^{T}\right]^{T} \Upsilon_{k} \tilde{x}_{k}^{(\mu)}\left(\tilde{x}_{k}^{(\mu)}\right)^{T} \Upsilon_{k}^{T}\right. \\
& \left.\times\left[\frac{1}{\sqrt{2}} \Delta_{g, k}^{T}, \frac{1}{\sqrt{2}} \Delta_{h, k}^{T}\right]\right\}\left[L_{g, k},-K_{k} L_{h, k}\right]^{T} \\
& +\sqrt{2} \mathbb{E}\left\{\check{A}_{k} e_{k}\left(\tilde{x}_{k}^{(\mu)}\right)^{T} \Upsilon_{k}^{T}\left[\frac{1}{\sqrt{2}} \Delta_{g, k}^{T}, \frac{1}{\sqrt{2}} \Delta_{h, k}^{T}\right]\right\} \\
& \times\left[L_{g, k},-K_{k} L_{h, k}\right]^{T}+\sqrt{2}\left[L_{g, k},-K_{k} L_{h, k}\right] \\
& \times \mathbb{E}\left\{\left[\frac{1}{\sqrt{2}} \Delta_{g, k}^{T}, \frac{1}{\sqrt{2}} \Delta_{h, k}^{T}\right]^{T} \Upsilon_{k} \tilde{x}_{k}^{(\mu)} e_{k}^{T} \check{A}_{k}^{T}\right\} \\
& +K_{k} \Psi_{k}^{w} K_{k}^{T}+\Psi_{k}^{v} .
\end{aligned}
$$

Proof: Substituting (28) and (30) into (33) yields

$$
\begin{aligned}
e_{k+1}= & \left(\left(A_{k}-K_{k} C_{k}\right)+\sqrt{2}\left[L_{g, k},-K_{k} L_{h, k}\right]\right. \\
& \left.\times\left[\frac{1}{\sqrt{2}} \Delta_{g, k}^{T}, \frac{1}{\sqrt{2}} \Delta_{h, k}^{T}\right]^{T} \Upsilon_{k}\right) e_{k}+v_{k}^{(\mu)}-K_{k} w_{k}^{(\mu)}
\end{aligned}
$$




$$
+\sqrt{2}\left[L_{g, k},-K_{k} L_{h, k}\right]\left[\frac{1}{\sqrt{2}} \Delta_{g, k}^{T}, \frac{1}{\sqrt{2}} \Delta_{h, k}^{T}\right]^{T} \Upsilon_{k} \tilde{x}_{k}^{(\mu)} .
$$

Noticing that $v_{k}^{(\mu)}$ and $w_{k}^{(\mu)}$ are zero-mean disturbances and independent of $e_{k},(39)$ can be obtained directly with the definition of $\check{A}_{k}$. The proof now is complete.

As discussed before, the accurate estimation error covariance in Lemma 4 could not be obtained due to the unknown matrix coefficients. To solve the estimation problem, an upper bound of the estimation error covariance will be derived in the next theorem, and the filter gain will then be designed to minimize the bound at each time step.

Theorem 1 Let $\gamma_{1, k}, \gamma_{2, k}, \gamma_{3, k}, \varepsilon$ be positive scalars. With initial condition $\bar{P}_{0}=P_{0}$, assume that the following discrete Riccati-like equation

$$
\begin{aligned}
\bar{P}_{k+1}= & (1+\varepsilon) A_{k}\left(\bar{P}_{k}^{-1}-\gamma_{1, k} \Upsilon_{k}^{T} \Upsilon_{k}\right)^{-1} A_{k}^{T}+2\left(\gamma_{1, k}^{-1}\right. \\
& \left.\times(1+\varepsilon)+\gamma_{2, k}^{-1}\left(1+\varepsilon^{-1}\right)\right) L_{g, k} L_{g, k}^{T}+\bar{\Psi}_{k}^{v} \\
& -Z_{k}^{T} Y_{k}^{-1} Z_{k}
\end{aligned}
$$

has positive definite solutions such that the following constraints

$$
\begin{aligned}
& \gamma_{1, k}^{-1} I-\Upsilon_{k} \bar{P}_{k} \Upsilon_{k}^{T}>0 \\
& \gamma_{2, k}^{-1} I-\Upsilon_{k} \tilde{x}_{k}^{(\mu)}\left(\tilde{x}_{k}^{(\mu)}\right)^{T} \Upsilon_{k}^{T}>0 \\
& \gamma_{3, k}^{-1} I-\Upsilon_{k} \bar{X}_{k} \Upsilon_{k}^{T}>0
\end{aligned}
$$

are satisfied, where

$$
\begin{aligned}
Y_{k}= & (1+\varepsilon) C_{k}\left(\bar{P}_{k}^{-1}-\gamma_{1, k} \Upsilon_{k}^{T} \Upsilon_{k}\right)^{-1} C_{k}^{T}+2\left(\gamma_{1, k}^{-1}\right. \\
& \left.\times(1+\varepsilon)+\gamma_{2, k}^{-1}\left(1+\varepsilon^{-1}\right)\right) L_{h, k} L_{h, k}^{T}+\bar{\Psi}_{k}^{w}, \\
Z_{k}= & (1+\varepsilon) C_{k}\left(\bar{P}_{k}^{-1}-\gamma_{1, k} \Upsilon_{k}^{T} \Upsilon_{k}\right)^{-1} A_{k}^{T}, \\
\bar{\Psi}_{a, b, k}^{v}= & \sum_{i=0}^{\mu} \sum_{j=0}^{\mu} \sum_{p=0}^{a} \sum_{q=0}^{b} \frac{1}{i ! j !} M_{a}^{p}\left(\left(\left(\nabla_{x}^{[i]} \otimes g^{[p]}\right) \bar{\Omega}_{i, j, k}\right.\right. \\
& \left.\times\left(\nabla_{x}^{[j]} \otimes g^{[q]}\right)^{T}\right) \otimes\left(\mathrm{st}_{n^{a-p}, n^{b-q}}^{-1}\left(\xi_{v, k, a-p+b-q}\right)\right. \\
& \left.\left.-\xi_{v, k, a-p} \xi_{v, k, b-q}^{T}\right)\right)\left(M_{b}^{q}\right)^{T}, \\
\bar{\Psi}_{a, b, k}^{w}= & \sum_{i=0}^{\mu} \sum_{j=0}^{\mu} \sum_{p=0}^{a} \sum_{q=0}^{b} \frac{1}{i ! j !} M_{a}^{p}\left(\left(\left(\nabla_{x}^{[i]} \otimes h^{[p]}\right) \bar{\Omega}_{i, j, k}\right.\right. \\
& \left.\times\left(\nabla_{x}^{[j]} \otimes h^{[q]}\right)^{T}\right) \otimes\left(\mathrm{st}_{n^{a-p}, n^{b-q}}^{-1}\left(\xi_{w, k, a-p+b-q}\right)\right.
\end{aligned}
$$

$$
\begin{aligned}
& \left.\left.-\xi_{w, k, a-p} \xi_{w, k, b-q}^{T}\right)\right)\left(M_{b}^{q}\right)^{T} \\
\bar{\Omega}_{i, j, k}= & \sum_{p=0}^{i} \sum_{q=0}^{j} M_{i}^{p}\left(\left(I_{n^{p}} \otimes\left(-\tilde{x}_{k}\right)^{[i-p]}\right) \bar{X}_{p, q, k}\right. \\
& \left.\left(I_{n^{q}} \otimes\left(-\tilde{x}_{k}\right)^{[j-q]}\right)^{T}\right)\left(M_{j}^{q}\right)^{T}, \\
\bar{X}_{k+1}= & A_{k}\left(\bar{X}_{k}^{-1}-\gamma_{3, k} \Upsilon_{k}^{T} \Upsilon_{k}\right)^{-1} A_{k}^{T}+\gamma_{3, k}^{-1} L_{g, k} L_{g, k}^{T} \\
& +\bar{\Psi}_{k}^{v}, \\
\bar{X}_{0}= & \mathbb{E}\left\{x_{0}^{(\mu)}\left(x_{0}^{(\mu)}\right)^{T}\right\} .
\end{aligned}
$$

Similar to $P_{k}$, matrices $\bar{X}_{k}, \bar{P}_{k}, \bar{\Psi}_{k}^{v}$ and $\bar{\Psi}_{k}^{w}$ are partitioned as follows:

$$
\begin{gathered}
\bar{X}_{k}=\left[\begin{array}{cccc}
\bar{X}_{0,0, k} & \bar{X}_{0,1, k} & \cdots & \bar{X}_{0, \mu, k} \\
\bar{X}_{1,0, k} & \bar{X}_{1,1, k} & \cdots & \bar{X}_{1, \mu, k} \\
\vdots & \vdots & \ddots & \vdots \\
\bar{X}_{\mu, 0, k} & \bar{X}_{\mu, 1, k} & \cdots & \bar{X}_{\mu, \mu, k}
\end{array}\right], \\
\bar{P}_{k}=\left[\begin{array}{cccc}
\bar{P}_{0,0, k} & \bar{P}_{0,1, k} & \cdots & \bar{P}_{0, \mu, k} \\
\bar{P}_{1,0, k} & \bar{P}_{1,1, k} & \cdots & \bar{P}_{1, \mu, k} \\
\vdots & \vdots & \ddots & \vdots \\
\bar{P}_{\mu, 0, k} & \bar{P}_{\mu, 1, k} & \cdots & \bar{P}_{\mu, \mu, k}
\end{array}\right], \\
\bar{\Psi}_{k}^{v}=\left[\begin{array}{cccc}
\bar{\Psi}_{0,0, k}^{v} & \bar{\Psi}_{0,1, k}^{v} & \cdots & \bar{\Psi}_{0, \mu, k}^{v} \\
\bar{\Psi}_{1,0, k}^{v} & \bar{\Psi}_{1,1, k}^{v} & \cdots & \bar{\Psi}_{1, \mu, k}^{v} \\
\vdots & \vdots & \ddots & \vdots \\
\bar{\Psi}_{\mu, 0, k}^{v} & \bar{\Psi}_{\mu, 1, k}^{v} & \cdots & \bar{\Psi}_{\mu, \mu, k}^{v}
\end{array}\right], \\
\bar{\Psi}_{k}^{w}=\left[\begin{array}{cccc}
\bar{\Psi}_{0,0, k}^{w} & \bar{\Psi}_{0,1, k}^{w} & \cdots & \bar{\Psi}_{0, \mu, k}^{w} \\
\bar{\Psi}_{1,0, k}^{w} & \bar{\Psi}_{1,1, k}^{w} & \cdots & \bar{\Psi}_{1, \mu, k}^{w} \\
\vdots & \vdots & \ddots & \vdots \\
\bar{\Psi}_{\mu, 0, k}^{w} & \bar{\Psi}_{\mu, 1, k}^{w} & \cdots & \bar{\Psi}_{\mu, \mu, k}^{w}
\end{array}\right],
\end{gathered}
$$

where $\bar{X}_{i, j, k}, \bar{P}_{i, j, k}, \bar{\Psi}_{i, j, k}^{v} \in \mathbb{R}^{n^{i} \times n^{j}}, \bar{\Psi}_{i, j, k}^{w} \in \mathbb{R}^{m^{i} \times m^{j}}$ for any $i, j=0,1, \ldots, \mu$. Then, with the filter gain given by

$$
K_{k}=Z_{k}^{T} Y_{k}^{-1},
$$

the matrix $\bar{P}_{k}$ is an upper bound of $P_{k}$ in the fault-free case. Moreover, the filter gain given by (52) minimizes the upper bound at each time step.

Proof: For notational simplicity, we denote

$$
\Psi_{i, j, k}^{v}=\mathbb{E}\left\{v_{i, k} v_{j, k}^{T}\right\}, \quad \Psi_{i, j, k}^{w}=\mathbb{E}\left\{w_{i, k} w_{j, k}^{T}\right\},
$$




$$
\begin{aligned}
X_{k} & =\mathbb{E}\left\{x_{k}^{(\mu)}\left(x_{k}^{(\mu)}\right)^{T}\right\}, \\
\Omega_{i, j, k} & =\mathbb{E}\left\{\left(x_{k}-\tilde{x}_{k}\right)^{[i]}\left(\left(x_{k}-\tilde{x}_{k}\right)^{[j]}\right)^{T}\right\} .
\end{aligned}
$$

$\Psi_{k}^{v}$ and $\Psi_{k}^{w}$ have been defined in Lemma 4.

Before proving that $\bar{P}_{k}$ is an upper bound of $P_{k}$, we are going to show that $X_{k} \leq \bar{X}_{k}, \Psi_{k}^{v} \leq \bar{\Psi}_{k}^{v}$, and $\Psi_{k}^{w} \leq$ $\bar{\Psi}_{k}^{w}$. These results could be obtained by induction. With $\bar{X}_{0}=\mathbb{E}\left\{x_{0}^{(\mu)}\left(x_{0}^{(\mu)}\right)^{T}\right\}$, we assume that $i=1,2, \ldots, k$, $X_{i} \leq \bar{X}_{i}$. Now it remains to show that $\Psi_{k}^{v} \leq \bar{\Psi}_{k}^{v}, \Psi_{k}^{w} \leq$ $\bar{\Psi}_{k}^{w}$, and $X_{k+1} \leq \bar{X}_{k+1}$.

Noting the fact that $v_{k}$ is independent of $x_{k}$, it follows from Lemma 1, (20) and (24) that

$$
\begin{aligned}
\Psi_{a, b, k}^{v}= & \sum_{i=0}^{\mu} \sum_{j=0}^{\mu} \sum_{p=0}^{a} \sum_{q=0}^{b} \frac{1}{i ! j !} M_{a}^{p}\left(\left(\left(\nabla_{x}^{[i]} \otimes g^{[p]}\right) \Omega_{i, j, k}\right.\right. \\
& \left.\times\left(\nabla_{x}^{[j]} \otimes g^{[q]}\right)^{T}\right) \otimes \mathbb{E}\left\{\left(v^{[a-p]}-\xi_{v, k, a-p}\right)\right. \\
& \left.\left.\times\left(v^{[b-q]}-\xi_{v, k, b-q}\right)^{T}\right\}\right)\left(M_{b}^{q}\right)^{T}
\end{aligned}
$$

and

$$
\begin{aligned}
\Omega_{i, j, k}= & \sum_{p=0}^{i} \sum_{q=0}^{j} M_{i}^{p}\left(( I _ { n ^ { p } } \otimes ( - \tilde { x } _ { k } ) ^ { [ i - p ] } ) \mathbb { E } \left\{x_{k}^{[p]}\right.\right. \\
& \left.\left.\times\left(x_{k}^{[q]}\right)^{T}\right\}\left(I_{n^{q}} \otimes\left(-\tilde{x}_{k}\right)^{[j-q]}\right)^{T}\right)\left(M_{j}^{q}\right)^{T} .
\end{aligned}
$$

In fact, $v_{m, k}$ can be written as:

$$
\begin{aligned}
v_{m, k}= & \sum_{i=0}^{\mu} \sum_{p=0}^{m} \frac{1}{i !} M_{m}^{p}\left(\left(\nabla_{x}^{[i]} \otimes g^{[p]}\right)\right. \\
& \left.\otimes\left(v_{k}^{[m-p]}-\xi_{v, k, m-p}\right)\right)\left(x_{k}-\tilde{x}_{k}\right)^{[i]} .
\end{aligned}
$$

Recalling Lemma 1 and the definition of $x_{k}^{(\mu)}$, one has

$$
v_{m, k}=\mathfrak{X}_{m}\left(\tilde{x}_{k}, u_{k}, v_{k}\right) x_{k}^{(\mu)},
$$

where $\mathfrak{X}_{m}(\cdot, \cdot, \cdot)$ is a proper nonlinear mapping. Then, $v_{k}^{(\mu)}$ can be expressed as

$$
v_{k}^{(\mu)}=\mathfrak{X}\left(\tilde{x}_{k}, u_{k}, v_{k}\right) x_{k}^{(\mu)},
$$

where

$$
\mathfrak{X}\left(\tilde{x}_{k}, u_{k}, v_{k}\right)=\left[\mathfrak{X}_{0}^{T}\left(\tilde{x}_{k}, u_{k}, v_{k}\right), \ldots, \mathfrak{X}_{\mu}^{T}\left(\tilde{x}_{k}, u_{k}, v_{k}\right)\right]^{T} .
$$

Now, it follows from the assumption $X_{k} \leq \bar{X}_{k}$ and the independence between $v_{k}$ and $x_{k}$ that

$$
\begin{aligned}
\Psi_{k}^{v} & =\mathbb{E}\left\{\mathfrak{X}\left(\tilde{x}_{k}, u_{k}, v_{k}\right) \mathbb{E}\left\{x_{k}^{(\mu)}\left(x_{k}^{(\mu)}\right)^{T}\right\} \mathfrak{X}^{T}\left(\tilde{x}_{k}, u_{k}, v_{k}\right)\right\} \\
& \leq \mathbb{E}\left\{\mathfrak{X}\left(\tilde{x}_{k}, u_{k}, v_{k}\right) \bar{X}_{k} \mathfrak{X}^{T}\left(\tilde{x}_{k}, u_{k}, v_{k}\right)\right\} .
\end{aligned}
$$

The above inequality shows that, by replacing the term $\mathbb{E}\left\{x_{k}^{[p]}\left(x_{k}^{[q]}\right)^{T}\right\}$ with $\bar{X}_{p, q, k}$ in (55), we would get an upper bound of $\Psi_{k}^{v}$. Therefore, from (47) and (49), it follows directly that $\Psi_{k}^{v} \leq \bar{\Psi}_{k}^{v}$. Similarly, we can get $\Psi_{k}^{w} \leq \bar{\Psi}_{k}^{w}$.

It remains to show that $X_{k+1} \leq \bar{X}_{k+1}$. With (28), we have

$$
\begin{aligned}
X_{k+1}= & \mathbb{E}\left\{\left(A_{k}+L_{g, k} \Delta_{g, k} \Upsilon_{k}\right) X_{k}\left(A_{k}+L_{g, k} \Delta_{g, k} \Upsilon_{k}\right)^{T}\right\} \\
& +\Psi_{k}^{v} .
\end{aligned}
$$

Since it has been proved that $\Psi_{k}^{v} \leq \bar{\Psi}_{k}^{v}$, we have

$$
\begin{aligned}
X_{k+1} \leq & \mathbb{E}\left\{\left(A_{k}+L_{g, k} \Delta_{g, k} \Upsilon_{k}\right) X_{k}\left(A_{k}+L_{g, k} \Delta_{g, k} \Upsilon_{k}\right)^{T}\right\} \\
& +\bar{\Psi}_{k}^{v} .
\end{aligned}
$$

With the assumption that $X_{k} \leq \bar{X}_{k}$ and the condition (44), it follows from Lemma 2 that

$$
\begin{aligned}
X_{k+1} & \leq A_{k}\left(\bar{X}_{k}^{-1}-\gamma_{3, k} \Upsilon_{k}^{T} \Upsilon_{k}\right)^{-1} A_{k}^{T}+\gamma_{3, k}^{-1} L_{g, k} L_{g, k}^{T} \\
& +\bar{\Psi}_{k}^{v}=\bar{X}_{k+1} .
\end{aligned}
$$

So far, we have proved that $X_{k} \leq \bar{X}_{k}, \Psi_{k}^{v} \leq \bar{\Psi}_{k}^{v}$, and $\Psi_{k}^{w} \leq \bar{\Psi}_{k}^{w}$, and we are going to deal with $P_{k}$ next. The corresponding results can also be obtained by induction. It is already known that $\bar{P}_{0}=P_{0}$. Then, assuming that, for $i=1,2, \ldots, k, P_{i} \leq \bar{P}_{i}$ and it remains to prove that $P_{k+1} \leq \bar{P}_{k+1}$.

With Lemma 3, we can have the following inequality:

$$
\begin{aligned}
& \sqrt{2} \check{A}_{k} e_{k}\left(\tilde{x}_{k}^{(\mu)}\right)^{T} \Upsilon_{k}^{T}\left[\frac{1}{\sqrt{2}} \Delta_{g, k}^{T}, \frac{1}{\sqrt{2}} \Delta_{h, k}^{T}\right] \\
& \times\left[L_{g, k},-K_{k} L_{h, k}\right]^{T}+\sqrt{2}\left[L_{g, k},-K_{k} L_{h, k}\right] \\
& \times\left[\frac{1}{\sqrt{2}} \Delta_{g, k}^{T}, \frac{1}{\sqrt{2}} \Delta_{h, k}^{T}\right]^{T} \Upsilon_{k} \tilde{x}_{k}^{(\mu)} e_{k}^{T} \check{A}_{k}^{T} \\
& \leq \varepsilon \check{A}_{k} e_{k} e_{k}^{T} \check{A}_{k}^{T}+2 \varepsilon^{-1}\left[L_{g, k},-K_{k} L_{h, k}\right]
\end{aligned}
$$




$$
\begin{aligned}
& \times\left[\frac{1}{\sqrt{2}} \Delta_{g, k}^{T}, \frac{1}{\sqrt{2}} \Delta_{h, k}^{T}\right]^{T} \Upsilon_{k} \tilde{x}_{k}^{(\mu)}\left(\tilde{x}_{k}^{(\mu)}\right)^{T} \Upsilon_{k}^{T} \\
& \times\left[\frac{1}{\sqrt{2}} \Delta_{g, k}^{T}, \frac{1}{\sqrt{2}} \Delta_{h, k}^{T}\right]\left[L_{g, k},-K_{k} L_{h, k}\right]^{T} .
\end{aligned}
$$

Substituting (64) into (39) yields

$$
\begin{aligned}
P_{k+1} \leq & (1+\varepsilon) \mathbb{E}\left\{\check{A}_{k} P_{k} \check{A}_{k}^{T}\right\}+2\left(1+\varepsilon^{-1}\right)\left[L_{g, k},-K_{k} L_{h, k}\right] \\
& \times \mathbb{E}\left\{\left[\frac{1}{\sqrt{2}} \Delta_{g, k}^{T}, \frac{1}{\sqrt{2}} \Delta_{h, k}^{T}\right]^{T} \Upsilon_{k} \tilde{x}_{k}^{(\mu)}\left(\tilde{x}_{k}^{(\mu)}\right)^{T} \Upsilon_{k}^{T}\right. \\
& \left.\times\left[\frac{1}{\sqrt{2}} \Delta_{g, k}^{T}, \frac{1}{\sqrt{2}} \Delta_{h, k}^{T}\right]\right\}\left[L_{g, k},-K_{k} L_{h, k}\right]^{T} \\
& +K_{k} \Psi_{k}^{w} K_{k}^{T}+\Psi_{k}^{v} .
\end{aligned}
$$

From the upper bounds of the covariances of $v_{k}^{(\mu)}$ and $w_{k}^{(\mu)}$ and the assumption that $P_{k} \leq \bar{P}_{k}$, we have

$$
\begin{aligned}
P_{k+1} \leq & (1+\varepsilon) \mathbb{E}\left\{\check{A}_{k} \bar{P}_{k} \check{A}_{k}^{T}\right\}+2\left(1+\varepsilon^{-1}\right)\left[L_{g, k},-K_{k} L_{h, k}\right] \\
& \times \mathbb{E}\left\{\left[\frac{1}{\sqrt{2}} \Delta_{g, k}^{T}, \frac{1}{\sqrt{2}} \Delta_{h, k}^{T}\right]^{T} \Upsilon_{k} \tilde{x}_{k}^{(\mu)}\left(\tilde{x}_{k}^{(\mu)}\right)^{T} \Upsilon_{k}^{T}\right. \\
& \left.\times\left[\frac{1}{\sqrt{2}} \Delta_{g, k}^{T}, \frac{1}{\sqrt{2}} \Delta_{h, k}^{T}\right]\right\}\left[L_{g, k},-K_{k} L_{h, k}\right]^{T} \\
& +K_{k} \bar{\Psi}_{k}^{w} K_{k}^{T}+\bar{\Psi}_{k}^{v} .
\end{aligned}
$$

Noticing that $\left\|\left[\frac{1}{\sqrt{2}} \Delta_{f, k}^{T}, \frac{1}{\sqrt{2}} \Delta_{h, k}^{T}\right]\right\| \leq 1$, it follows from (42), (43) and Lemma 2 that

$$
\begin{aligned}
P_{k+1} \leq & (1+\varepsilon)\left(\left(A_{k}-K_{k} C_{k}\right)\left(\bar{P}_{k}^{-1}-\gamma_{1, k} \Upsilon_{k}^{T} \Upsilon_{k}\right)^{-1}\right. \\
& \times\left(A_{k}-K_{k} C_{k}\right)^{T}+2 \gamma_{1, k}^{-1}\left(L_{g, k} L_{g, k}^{T}+K_{k} L_{h, k}\right. \\
& \left.\left.\times L_{h, k}^{T} K_{k}^{T}\right)\right)+2 \gamma_{2, k}^{-1}\left(1+\varepsilon^{-1}\right)\left(L_{g, k} L_{g, k}^{T}\right. \\
& \left.+K_{k} L_{h, k} L_{h, k}^{T} K_{k}^{T}\right)+\bar{\Psi}_{k}^{v}+K_{k} \bar{\Psi}_{k}^{w} K_{k}^{T} .
\end{aligned}
$$

Furthermore, it can be seen from (45) and (46) that

$$
\begin{aligned}
P_{k+1} \leq & K_{k} Y_{k} K_{k}^{T}-K_{k} Z_{k}-Z_{k}^{T} K_{k}^{T}+(1+\varepsilon) A_{k}\left(\bar{P}_{k}^{-1}\right. \\
& \left.-\gamma_{1, k} \Upsilon_{k}^{T} \Upsilon_{k}\right)^{-1} A_{k}^{T}+2\left(\gamma_{1, k}^{-1}(1+\varepsilon)+\gamma_{2, k}^{-1}(1\right. \\
& \left.\left.+\varepsilon^{-1}\right)\right) L_{g, k} L_{g, k}^{T}+\bar{\Psi}_{k}^{v} .
\end{aligned}
$$

Considering $Y_{k}=Y_{k}^{T}>0$ and completing the square with respect to $K_{k}$, we have

$$
\begin{aligned}
P_{k+1} \leq & \left(K_{k}-Z_{k}^{T} Y_{k}^{-1}\right) Y_{k}\left(K_{k}-Z_{k}^{T} Y_{k}^{-1}\right)^{T}-Z_{k}^{T} Y_{k}^{-1} Z_{k} \\
& +2\left(\gamma_{1, k}^{-1}(1+\varepsilon)+\gamma_{2, k}^{-1}\left(1+\varepsilon^{-1}\right)\right) L_{g, k} L_{g, k}^{T} \\
& +(1+\varepsilon) A_{k}\left(\bar{P}_{k}^{-1}-\gamma_{1, k} \Upsilon_{k}^{T} \Upsilon_{k}\right)^{-1} A_{k}^{T}+\bar{\Psi}_{k}^{v} .
\end{aligned}
$$

It is now obvious that, when $K_{k}=Z_{k}^{T} Y_{k}^{-1}$, the upper bound of $P_{k+1}$ is minimized and

$$
\begin{aligned}
P_{k+1} \leq & -Z_{k}^{T} Y_{k}^{-1} Z_{k}+(1+\varepsilon) A_{k}\left(\bar{P}_{k}^{-1}-\gamma_{1, k} \Upsilon_{k}^{T} \Upsilon_{k}\right)^{-1} \\
& \times A_{k}^{T}+2\left(\gamma_{1, k}^{-1}(1+\varepsilon)+\gamma_{2, k}^{-1}\left(1+\varepsilon^{-1}\right)\right) L_{g, k} L_{g, k}^{T} \\
& +\bar{\Psi}_{k}^{v}=\bar{P}_{k+1},
\end{aligned}
$$

which concludes the proof.

Remark 5 The filter gain $K_{k}$ is calculated at each time instant to minimize an upper bound of the filtering error covariance. The system (28) under consideration includes the polynomial approximation errors, thereby better reflecting the reality. Specific efforts have been made to handle the approximation errors. Though the unknown matrices $\Delta_{g, k}$ and $\Delta_{h, k}$ can not be introduced to design the filter, the matrices $L_{g, k}$ and $L_{h, k}$ reflect the effects of the approximation errors on the filter design in a quantitative way. The value of the state estimate $\tilde{x}_{k}^{(\mu)}$ can be directly used to design $K_{k}$ since $\tilde{x}_{k}^{(\mu)}$ is already obtained at time step $k$. Due to the consideration of the polynomial approximation errors, the accurate covariances $\Psi_{k}^{v}$ and $\Psi_{k}^{w}$ cannot be obtained as done in $[14,15]$. Instead, the upper bounds of the covariances have been calculated and employed to design the suboptimal filter. It is worth mentioning that, $\bar{\Phi}_{k}^{w}$, an upper bound of $\mathbb{E}\left\{w_{k}^{(\mu)}\left(w_{k}^{(\mu)}\right)^{T}\right\}$, could guarantee the invertibility of $Y_{k}$ in (52). The parameter $\varepsilon$ can be determined to balance the intrinsic characteristic of the proposed polynomial filter and the impact brought from the state estimates in the upper bound of the filtering error covariance. The proposed algorithm can be applied to nonlinear time-varying systems that are $\mu+1$ times continuously differentiable. Furthermore, the gain $K_{k}$ can be designed recursively by a Riccati-like equation, which is applicable for online computations.

Theorem 1 provides a recursive way to obtain the filter gain $K_{k}$. The algorithm is summarized in Algorithm 1 to show the calculation of $K_{k}$ at each time step with a given order $\mu$.

\section{Fault Detection}

To efficiently detect the fault in the system (1), we need to define a residual signal based on the proposed polynomial filter and then evaluate it properly. Here, we utilize the following signal as the residual:

$$
r_{k}=y_{k}^{(\mu)}-C_{k} \tilde{x}_{k}^{(\mu)} .
$$

In the next theorem, a time-varying fault detection threshold will be calculated for the residual defined in (71). The state estimate, approximation error and bounds of the noises are all taken into consideration. 


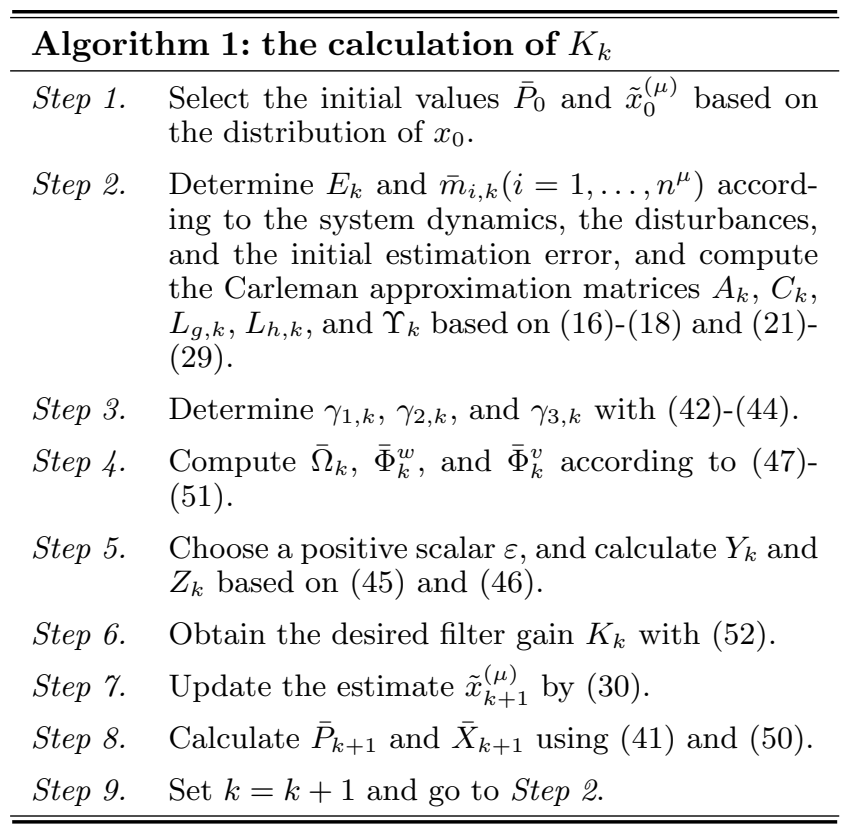

Theorem 2 For system (1), let us consider the filter (30) designed in Theorem 1. Then, the residual in (71) satisfies the following inequality in the fault free case:

$$
\begin{aligned}
\left\|r_{k}\right\| \leq & \left(\left\|C_{k}\right\|+\left\|L_{h, k}\right\|\left\|\Upsilon_{k}\right\|\right) \delta_{e, k}+\left\|L_{h, k}\right\|\left\|\Upsilon_{k} \tilde{x}_{k}^{(\mu)}\right\| \\
& +\delta_{w, k}:=\delta_{r, k}
\end{aligned}
$$

where

$$
\begin{aligned}
& \delta_{e, k+1}=\left(\left\|A_{k}-K_{k} C_{k}\right\|+\sqrt{2}\left\|\left[L_{g, k},-K_{k} L_{h, k}\right]\right\|\left\|\Upsilon_{k}\right\|\right) \\
& \times \delta_{e, k}+\sqrt{2}\left\|\left[L_{g, k},-K_{k} L_{h, k}\right]\right\|\left\|\Upsilon_{k} \tilde{x}_{k}^{(\mu)}\right\| \\
& +\left\|K_{k}\right\| \delta_{w, k}+\delta_{v, k} \text {, } \\
& \delta_{v, m, k}=\sum_{i=0}^{\mu} \sum_{p=0}^{m} \frac{1}{i !}\left\|M_{m}^{p}\right\|\left\|\nabla_{x}^{[i]} \otimes g^{[p]}\right\| \bar{\rho}_{i, k}\left(s_{v, k, m-p}\right. \\
& \left.+\left\|\xi_{v, k, m-p}\right\|\right) \text {, } \\
& \delta_{w, m, k}=\sum_{i=0}^{\mu} \sum_{p=0}^{m} \frac{1}{i !}\left\|M_{m}^{p}\right\|\left\|\nabla_{x}^{[i]} \otimes h^{[p]}\right\| \bar{\rho}_{i, k}\left(s_{w, k, m-p}\right. \\
& \left.+\left\|\xi_{w, k, m-p}\right\|\right) \text {, } \\
& \bar{\rho}_{i, k}=\sum_{q=0}^{i}\left\|M_{i}^{q}\left(I_{n^{q}} \otimes\left(-\tilde{x}_{k}\right)^{[i-q]}\right)\right\|\left(\left\|\tilde{x}_{q, k}^{(\mu)}\right\|+\delta_{e, k}\right), \\
& \delta_{v, k}=\sqrt{\sum_{m=0}^{\mu}\left(\delta_{v, m, k}\right)^{2}} \\
& \delta_{w, k}=\sqrt{\sum_{m=0}^{\mu}\left(\delta_{w, m, k}\right)^{2}}
\end{aligned}
$$

and the initial values of $\delta_{e, k}$ are given by

$$
\delta_{e, i, 0}=s_{0, i}+\left\|\zeta_{0, i}\right\|, \delta_{e, 0}=\sqrt{\sum_{i=0}^{\mu} \delta_{e, i, 0}^{2}}
$$

Then $\delta_{r, k}$ is the threshold that we can utilize to detect the fault.

Proof: Substituting (28) and (30) into (71), we have

$$
r_{k}=C_{k} e_{k}+L_{h, k} \Delta_{h, k} \Upsilon_{k}\left(e_{k}+\tilde{x}_{k}^{(\mu)}\right)+w_{k}^{(\mu)}
$$

With $\left\|\Delta_{h, k}\right\| \leq 1$ and triangle inequality, we can get

$$
\begin{aligned}
\left\|r_{k}\right\| \leq & \left(\left\|C_{k}\right\|+\left\|L_{h, k}\right\|\left\|\Upsilon_{k}\right\|\right)\left\|e_{k}\right\|+\left\|L_{h, k}\right\|\left\|\Upsilon_{k} \tilde{x}_{k}^{(\mu)}\right\| \\
& +\left\|w_{k}^{(\mu)}\right\| .
\end{aligned}
$$

If we can prove that $\left\|e_{k}\right\| \leq \delta_{e, k}$ and $\left\|w_{k}^{(\mu)}\right\| \leq \delta_{w, k}$, then (72) follows directly from (81).

It follows from (25) that,

$$
\begin{aligned}
\left\|w_{m, k}^{(\mu)}\right\| \leq & \sum_{i=0}^{\mu} \sum_{p=0}^{m} \frac{1}{i !}\left\|M_{m}^{p}\right\|\left\|\nabla_{x}^{[i]} \otimes h^{[p]}\right\|\left\|\left(x_{k}-\tilde{x}_{k}\right)^{[i]}\right\| \\
& \times\left\|w_{k}^{[m-p]}-\xi_{w, k, m-p}\right\|
\end{aligned}
$$

and the upper bound of $\left\|\left(x_{k}-\tilde{x}_{k}\right)^{[i]}\right\|$ can be determined as follows:

$$
\begin{aligned}
\left\|\left(x_{k}-\tilde{x}_{k}\right)^{[i]}\right\| & =\left\|\sum_{q=0}^{i} M_{i}^{q}\left(I_{n^{q}} \otimes\left(-\tilde{x}_{k}\right)^{[i-q]}\right) x_{k}^{[q]}\right\| \\
\leq & \sum_{q=0}^{i}\left\|M_{i}^{q}\left(I_{n^{q}} \otimes\left(-\tilde{x}_{k}\right)^{[i-q]}\right)\right\| \\
& \times\left\|\tilde{x}_{q, k}^{(\mu)}+e_{q, k}\right\| .
\end{aligned}
$$

Noticing the fact that $\left\|e_{q, k}\right\| \leq\left\|e_{k}\right\|$, we have

$$
\begin{aligned}
\left\|\left(x_{k}-\tilde{x}_{k}\right)^{[i]}\right\| \leq & \sum_{q=0}^{i}\left\|M_{i}^{q}\left(I_{n^{q}} \otimes\left(-\tilde{x}_{k}\right)^{[i-q]}\right)\right\| \\
\leq & \sum_{q=0}^{i}\left\|M_{i}^{q}\left(I_{n^{q}} \otimes\left(-\tilde{x}_{k}\right)^{[i-q]}\right)\right\| \\
& \times\left(\left\|e_{k}\right\|+\left\|\tilde{x}_{k}^{(\mu)}\right\|\right) .
\end{aligned}
$$

With (84), we can see that if $\left\|e_{k}\right\| \leq \delta_{e, k}$, then $\left\|w_{m, k}^{(\mu)}\right\| \leq$ $\delta_{w, m, k}$ and $\left\|w_{k}^{(\mu)}\right\| \leq \delta_{w, k}$. Similarly, we have that if $\left\|e_{k}\right\| \leq \delta_{e, k}$, then $\left\|v_{k}^{(\mu)}\right\| \leq \delta_{v, k}$. Thus, to obtain (72), 
all we need to verify is that $\left\|e_{k}\right\| \leq \delta_{e, k}$. This can be proved by induction. It is obvious that $\left\|e_{0}\right\| \leq \delta_{e, 0}$. Assuming that $\left\|e_{i}\right\| \leq \delta_{e, i}(i=1,2, \ldots, k)$, we are going to demonstrate that $\left\|e_{k+1}\right\| \leq \delta_{e, k+1}$.

With (40) and $\left\|\left[\frac{1}{\sqrt{2}} \Delta_{f, k}^{T}, \frac{1}{\sqrt{2}} \Delta_{h, k}^{T}\right]\right\| \leq 1$, it follows that

$$
\begin{aligned}
\left\|e_{k+1}\right\| \leq & \left(\left\|A_{k}-K_{k} C_{k}\right\|+\sqrt{2}\left\|\left[L_{g, k},-K_{k} L_{h, k}\right]\right\|\left\|\Upsilon_{k}\right\|\right) \\
& \times\left\|e_{k}\right\|+\sqrt{2}\left\|\left[L_{g, k},-K_{k} L_{h, k}\right]\right\|\left\|\Upsilon_{k} \tilde{x}_{k}^{(\mu)}\right\| \\
& +\left\|v_{k}^{(\mu)}\right\|+\left\|K_{k}\right\|\left\|w_{k}^{(\mu)}\right\| .
\end{aligned}
$$

Based on our assumption that $\left\|e_{k}\right\| \leq \delta_{e, k}$, we have $\left\|w_{k}^{(\mu)}\right\| \leq \delta_{w, k}$ and $\left\|v_{k}^{(\mu)}\right\| \leq \delta_{v, k}$. Then it is straightforward to see that $\left\|e_{k+1}\right\| \leq \delta_{e, k+1}$. Now we can draw the conclusion that $\left\|e_{k}\right\| \leq \delta_{e, k}$. Substituting $\left\|e_{k}\right\| \leq \delta_{e, k}$ and $\left\|w_{k}^{(\mu)}\right\| \leq \delta_{w, k}$ into (81) yields (72). The proof now is complete.

The algorithm is summarized in Algorithm 2 to show the determination of the threshold $\delta_{r, k}$ at each time step with a given order $\mu$. Based on Theorem 2, a natural

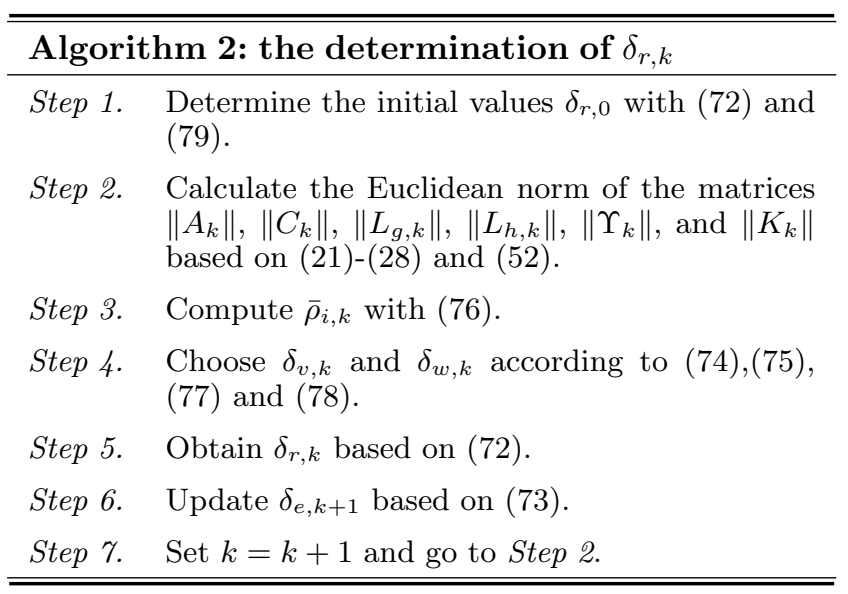

fault detection strategy follows directly as follows:

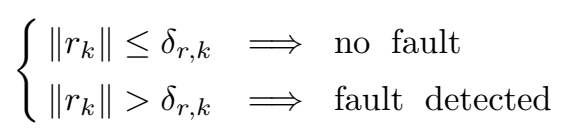

With the proposed filter and fault detection threshold, the fault detectability is analyzed in the sequel.

Theorem 3 For system (1), consider the filter (30) designed in Theorem 1 and the fault detection strategy given in (86). The fault will be detected at time step $k+1$ if the following inequality holds:

$$
\begin{aligned}
\alpha_{v, f, k}> & \frac{1}{\left\|C_{k+1}\right\|-\left\|L_{f, h, k+1}\right\|\left\|\Upsilon_{k+1}\right\| \|}\left(\delta_{r, k+1}+\delta_{w, k+1}\right. \\
& \left.+\left\|L_{f, h, k+1}\right\|\left\|\Upsilon_{k+1} \tilde{x}_{k+1}^{(\mu)}\right\|\right)+\left\|K_{k}\right\|\left\|w_{k}^{(\mu)}\right\|
\end{aligned}
$$

$$
\begin{aligned}
& +\left(\left\|A_{k}-K_{k} C_{k}\right\|+\sqrt{2}\left\|\left[L_{f, g, k},-K_{k} L_{f, h, k}\right]\right\|\right. \\
& \left.\times\left\|\Upsilon_{k}\right\|\right) \delta_{e, k}+\sqrt{2}\left\|\left[L_{f, g, k},-K_{k} L_{f, h, k}\right]\right\| \\
& \times\left\|\Upsilon_{k} \tilde{x}_{k}^{(\mu)}\right\|,
\end{aligned}
$$

where

$$
\begin{aligned}
\alpha_{v, f, m, k}= & \left(\left\|f_{k}\right\|-s_{v, k, 1}\right)^{m}-\left\|\xi_{v, k, m}\right\| \\
& -\sum_{i=0}^{\mu} \sum_{p=1}^{m} \frac{1}{i !}\left\|M_{m}^{p}\left(\nabla_{x}^{[i]} \otimes g^{[p]}\right)\right\| \bar{\rho}_{i, k}\left(\left(\left\|f_{k}\right\|\right.\right. \\
& \left.\left.+s_{v, k, 1}\right)^{m-p}+\left\|\xi_{v, k, m-p}\right\|\right) \\
\alpha_{v, f, k}= & \sqrt{\sum_{m=0}^{\mu}\left(\alpha_{v, f, m, k}\right)^{2}}
\end{aligned}
$$

$L_{f, g, k}$ and $L_{f, h, k}$ are the problem-dependent matrices in the faulty case, which are corresponding to $L_{g, k}$ and $L_{h, k}$ in the fault-free case, respectively.

Proof: In the presence of $f_{k}, v_{m, k}$ can be written as

$$
\begin{aligned}
v_{m, k}= & \sum_{i=0}^{\mu} \sum_{p=0}^{m} \frac{1}{i !} M_{m}^{p}\left(\left(\left(\nabla_{x}^{[i]} \otimes g^{[p]}\right)\left(x_{k}-\tilde{x}_{k}\right)^{[i]}\right)\right. \\
& \left.\otimes\left(\left(v_{k}+f_{k}\right)^{[m-p]}-\xi_{v, k, m-p}\right)\right) .
\end{aligned}
$$

Then it follows directly that

$$
\begin{aligned}
\left\|v_{m, k}\right\| \geq & \left(\left\|f_{k}\right\|-s_{v, k, 1}\right)^{m}-\left\|\xi_{v, k, m}\right\| \\
& -\sum_{i=0}^{\mu} \sum_{p=1}^{m} \frac{1}{i !}\left\|M_{m}^{p}\left(\nabla_{x}^{[i]} \otimes g^{[p]}\right)\right\| \bar{\rho}_{i, k}\left(\left(\left\|f_{k}\right\|\right.\right. \\
& \left.\left.+s_{v, k, 1}\right)^{m-p}+\left\|\xi_{v, k, m-p}\right\|\right)=\alpha_{v, f, m, k} .
\end{aligned}
$$

Subsequently, we have

$$
\left\|v_{k}^{(\mu)}\right\| \geq \alpha_{v, f, k} .
$$

With (40), we have

$$
\begin{aligned}
\left\|e_{k+1}\right\| \geq & \left\|v_{k}^{(\mu)}\right\|-\sqrt{2}\left\|\left[L_{f, g, k},-K_{k} L_{f, h, k}\right]\right\|\left\|\Upsilon_{k} \tilde{x}_{k}^{(\mu)}\right\| \\
& -\left(\left\|A_{k}-K_{k} C_{k}\right\|+\sqrt{2}\left\|\left[L_{f, g, k},-K_{k} L_{f, h, k}\right]\right\|\right. \\
& \left.\times\left\|\Upsilon_{k}\right\|\right)\left\|e_{k}\right\|-\left\|K_{k}\right\|\left\|w_{k}^{(\mu)}\right\| .
\end{aligned}
$$

Substituting (87) into (93) yields

$$
\begin{aligned}
\left\|e_{k+1}\right\|> & \frac{1}{\left\|C_{k+1}\right\|-\left\|L_{f, h, k+1}\right\|\left\|\Upsilon_{k+1}\right\| \mid}\left(\delta_{r, k+1}\right. \\
& \left.+\delta_{w, k+1}+\left\|L_{f, h, k+1}\right\|\left\|\Upsilon_{k+1} \tilde{x}_{k+1}^{(\mu)}\right\|\right) .
\end{aligned}
$$


Considering (80), we have

$$
\begin{array}{r}
\left\|r_{k+1}\right\| \geq\left|\left\|C_{k+1}\right\|-\left\|L_{f, h, k+1}\right\|\left\|\Upsilon_{k+1}\right\|\right|\left\|e_{k+1}\right\| \\
-\left\|L_{f, h, k+1}\right\|\left\|\Upsilon_{k+1} \tilde{x}_{k+1}^{(\mu)}\right\|-\left\|w_{k+1}^{(\mu)}\right\| .
\end{array}
$$

From (94) and (95), it follows that

$$
\left\|r_{k+1}\right\| \geq \delta_{r, k+1} .
$$

Based on the proposed fault detection strategy (86), the fault can be detected at time step $k+1$ and that concludes the proof.

Remark 6 Theorem 2 puts forward a recursive method to determine the fault detection threshold for system (28). With the adaptive threshold, abnormal changes in systems can be detected accordingly. The fault may be some state-dependent abrupt changes (e.g. actuator or sensor failures in a control system). The approximation errors, additive disturbances, and initial estimation error have all been considered in the threshold. The spectral norms of matrices and the upper bounds of Euclidean norms of vector disturbances have been introduced to calculate the threshold. In this way, the false alarms can be avoided in the proposed fault detection scheme. Moreover, the threshold is related to the measurement and estimated states, and hence needs to be updated online at each time step, which makes the method adaptive. With the proposed filter and fault detection strategy, the fault detectability is analyzed in Theorem 3 as well. The fault detectability condition established in Theorem 3 takes the Carleman approximation and the approximation error into account and therefore looks complicated. Nevertheless, such a condition can be easily verified and also provides inequality constraints on the fault size. The explicit consideration of the approximation error in the filter and fault detector designs constitutes the main difference between our work and those in [14,15], and the derivation of the threshold and fault detectability analysis for nonlinear systems with polynomial approximation are new. If the stability analysis of the proposed filter approach becomes a concern, some additional assumptions can be made on the system parameters so as to ensure the boundedness of the estimation errors [25, 32]. In the next section, a numerical simulation would be carried out to show the effectiveness of the presented fault detection strategy.

\section{Illustrative Example}

Consider the following nonlinear discrete system:

$$
\left\{\begin{aligned}
x_{1, k+1} & =0.85 x_{1, k}+0.5 x_{2, k} \sin \left(x_{1, k}\right)+v_{1, k}+f_{k}, \\
x_{2, k+1} & =1.15 u_{k}-0.5 x_{1, k} \sin \left(x_{2, k}\right)+v_{2, k}, \\
y_{k} & =x_{2, k}+w_{k}, \\
u_{k} & =y_{k} .
\end{aligned}\right.
$$

The state noises and measurement noise are independent and obey the following distributions (for $i=1,2$ ):

$$
\left\{\begin{array}{l}
P\left(v_{i, k}=-1 \times 10^{-3}\right)=0.6 \\
P\left(v_{i, k}=0\right)=0.2 \\
P\left(v_{i, k}=3 \times 10^{-3}\right)=0.2
\end{array}\right.
$$

and

$$
\left\{\begin{array}{l}
P\left(w_{k}=-7 \times 10^{-3}\right)=0.3 \\
P\left(w_{k}=3 \times 10^{-3}\right)=0.7 .
\end{array}\right.
$$

When the order $\mu=2$, how to construct the polynomial filter is explained step by step as follows. For the addressed system, we have

$$
\left\{\begin{aligned}
g\left(x_{k}, u_{k}\right) & =\left[\begin{array}{c}
0.85 x_{1, k}+0.5 x_{2, k} \sin \left(x_{1, k}\right) \\
1.15 u_{k}-0.5 x_{1, k} \sin \left(x_{2, k}\right)
\end{array}\right] \\
h\left(x_{k}\right) & =x_{2, k}
\end{aligned}\right.
$$

With the linear measurement, we can easily obtain that $L_{h, k}=0$ and

$$
C_{k}=\left[\begin{array}{ccccccc}
1 & 0 & 0 & 0 & 0 & 0 & 0 \\
0 & 0 & 1 & 0 & 0 & 0 & 0 \\
\mathbb{E}\left\{w_{k}^{[2]}\right\} & 0 & 0 & 0 & 0 & 0 & 1
\end{array}\right]
$$

With (22) and (29), $A_{k}$ can be determined. Based on (26), we have

$$
\Upsilon_{k}=\left[\begin{array}{ccccccc}
\tilde{x}_{1, k}^{2} & -2 \tilde{x}_{1, k} & 0 & 1 & 0 & 0 & 0 \\
\tilde{x}_{1, k} \tilde{x}_{2, k} & -\tilde{x}_{2, k} & -\tilde{x}_{1, k} & 0 & 1 & 0 & 0 \\
\tilde{x}_{1, k} \tilde{x}_{2, k} & -\tilde{x}_{2, k} & -\tilde{x}_{1, k} & 0 & 0 & 1 & 0 \\
\tilde{x}_{2, k}^{2} & 0 & -2 \tilde{x}_{2, k} & 0 & 0 & 0 & 1
\end{array}\right] .
$$

The matrix $E_{k}$ is set to be $0.075 I$ based on the system dynamics, the disturbances, and the initial estimation error, and it can be seen that the assumption on the estimation error (i.e. $x_{k}=\tilde{x}_{k}+E_{k} z_{k}$ for some $z_{k} \in \mathbb{R}^{n}$ and $\left\|z_{k}\right\| \leq 1$ ) holds with such a choice of $E_{k}$. Based on $E_{k}$ and the values of $\bar{m}_{i, k}$ for $i=1,2, \ldots, n^{\mu}$, the matrix $L_{g, k}$ can be chosen as $0.1 I$. After getting all the aforementioned parameters, Theorem 1 can be applied to recursively calculate the desired filter gain.

In the fault-free case, the average estimation error covariances with the proposed method and the ones with 
the conventional PEKF algorithm [14,15] are both listed in Table 1. Monte-Carlo simulations with 50 runs are carried out in the cases where $\mu=1$ and $\mu=2$. From the table, two conclusions can be drawn: 1) approximating the system with a polynomial of a higher order implies a more accurate approximation of the original nonlinear system and gives better estimation results, no matter whether the approximation errors are considered or not; and 2) the proposed filter outperforms classical PEKF, thanks to the specific efforts we have made to deal with the high-order approximation errors.

Table 1

Average Trace of the Estimation Error Covariance

\begin{tabular}{|c|c|c|}
\hline & $\mu=1$ & $\mu=2$ \\
\hline Traditional PEKF & $6.1763 \times 10^{-4}$ & $4.9531 \times 10^{-4}$ \\
\hline Proposed Filter & $5.5649 \times 10^{-4}$ & $3.9534 \times 10^{-4}$ \\
\hline
\end{tabular}

To demonstrate the fault detection strategy, we add the following fault to the system:

$$
f_{k}=\left\{\begin{array}{cc}
0.003, & \text { if } k>20 \\
0, & \text { otherwise }
\end{array}\right.
$$

Figs. 1-2 show the actual states and the estimates when $\mu=2$. It can be concluded that, the proposed filter performs well when there is no fault in the system (when $k \leq 20$ ), and the obvious difference between the actual state and estimated state in the faulty case (when $k>$ 20) could help us to detect the fault. The Euclidean norm of residual and the adaptive threshold are illustrated in Fig. 3. It can be seen that using the adaptive threshold, the additive fault could be detected immediately after it occurs. This result presents the effectiveness of our adaptive fault detection strategy.

Remark 7 The computational complexity of the proposed algorithm is $O\left(n^{3 \mu}\right)$. The numerical experiments are performed on a $2.83 \mathrm{GHz}$ quad-core desktop computer with 3.50 GB RAM using 32 bit MATLAB. When $\mu=1$, the execution time is $15 \mathrm{~ms}$; when $\mu=2$, the execution time is $94 \mathrm{~ms}$. So it is obvious that the computational burden of the proposed algorithm increases exponentially with a bigger degree of polynomials. When $\mu=2$, the average Euclidean norm of the residuals obtained with the proposed filter after the fault occurs is $7.250 \times 10^{-3}$ in 50 Monte-Carlo simulations. With traditional PEKF, the average Euclidean norm of the residuals is $5.280 \times 10^{-3}$. So the proposed filter are more sensitive to the fault and can better solve the fault detection problem.

\section{Conclusion}

The filtering and fault detection problems for a class of nonlinear systems have been addressed with a polynomial approach in the paper. The nonlinear functions

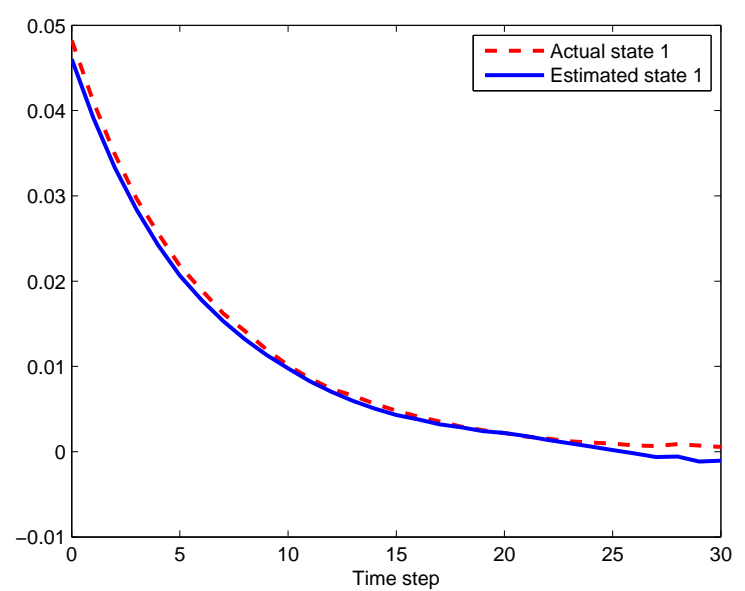

Fig. 1. The actual state $x_{1}$ and its estimate

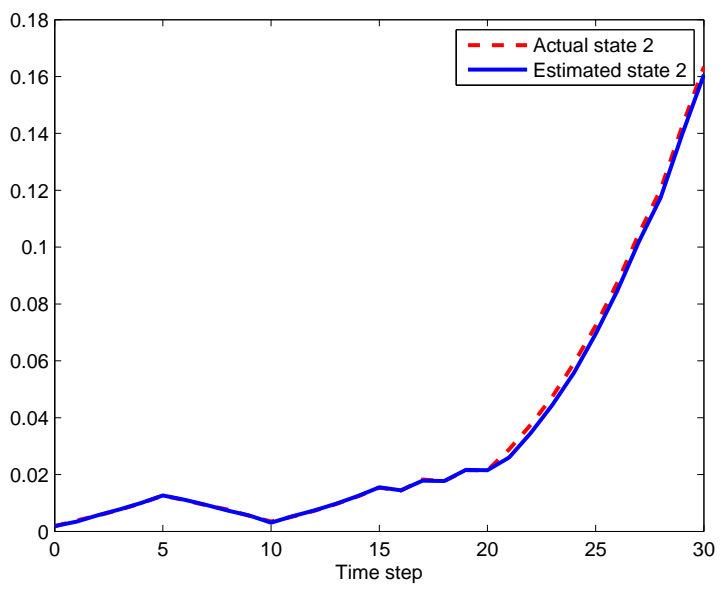

Fig. 2. The actual state $x_{2}$ and its estimate

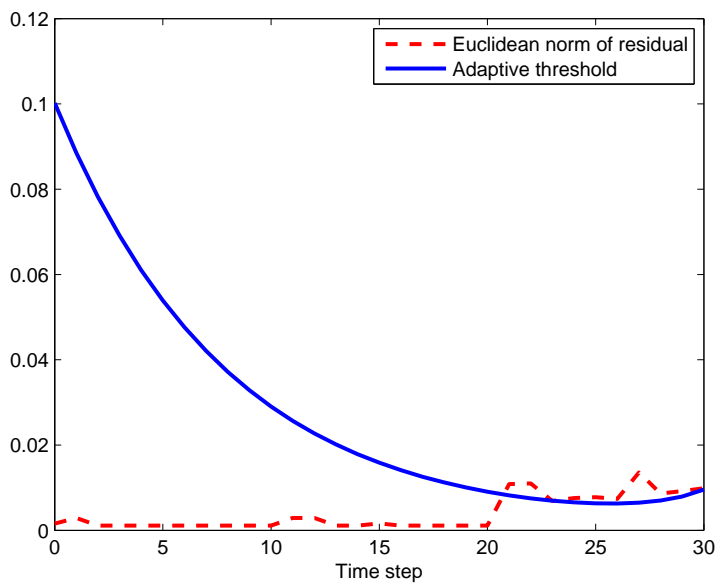

Fig. 3. The Euclidean norm of residual and the threshold 
have been approximated with polynomials of a chosen degree, and the approximation errors, which result from high-order remainder terms of series expansions, have been written as low-order terms with norm-bounded coefficients. An upper bound of the filtering error covariance has been derived in the presence of the approximation errors. Then the filter has been designed to minimize the bound at each time step in the fault-free case. The desired filter gain could be obtained with a set of Riccati-like recursive matrix equations, thus the algorithm is applicable for online computation. A fault detection strategy with adaptive threshold has been proposed to efficiently detect the possible fault, taking account of the noises and the approximation errors. Sufficient conditions have been established to guarantee the fault detectability for the proposed fault detection scheme. A numerical simulation has been given to illustrate that the proposed method could achieve accurate state estimation and effective fault detection. It should be noted that two of the future research topics would be to investigate the fault diagnosis and fault tolerant control problems after the fault detection.

\section{Acknowledgements}

This work was supported by the the National Natural Science Foundation of China under grants 61490701, 61210012, 61290324, and 61273156, and Jiangsu Provincial Key Laboratory of E-business at Nanjing University of Finance and Economics of China under Grant JSEB201301.

\section{References}

[1] M. Basin. New trends in optimal filtering and control for polynomial and time-delay systems. Springer-Verlag Berlin Heidelberg, 2008.

[2] M. Basin, S. Elvira-Ceja, and E. Sanchez. Mean-square $\mathcal{H}_{\infty}$ filtering for stochastic systems: Application to a $2 \mathrm{DOF}$ helicopter. Signal Processing, 92(3): 801-806, 2012.

[3] M. Basin, and P. Rodriguez-Ramirez. Sliding mode filter design for nonlinear polynomial systems with unmeasured states. Information Sciences, 204: 82-91, 2012.

[4] M. Basin, P. Shi, and D. Calderon-Alvarez. Central suboptimal $\mathcal{H}_{\infty}$ filter design for nonlinear polynomial systems. International Journal of Adaptive Control and Signal Processing, 23(10): 926-939, 2009.

[5] M. Basin, P. Shi, and D. Calderon-Alvarez. Approximate finite-dimensional filtering for polynomial states over polynomial observations. International Journal of Control, 83(4): 724-730, 2010

[6] R. Bellman. Introduction to Matrix Analysis. New York: McGraw-Hill, 1970.

[7] R. Caballero-Águila, A. Hermoso-Carazo, and J. LinaresPérez. Extended and unscented filtering algorithms in nonlinear fractional order systems with uncertain observations. Applied Mathematical Sciences, 6(30): 14711486,2012
[8] G. Calafiore. Reliable localization using set-valued nonlinear filters. IEEE Transactions on Systems, Man and Cybernetics, Part A: Systems and Humans, 35(2): 189-197, 2005.

[9] F. Carravetta, A. Germani, and M. Raimondi. Polynomial filtering for linear discrete time non-Gaussian systems. SIAM Journal on Control and Optimization, 34(5): 1666-1690, 1996.

[10] F. Carravetta, A. Germani, and M. Raimondi. Polynomial filtering of discrete-time stochastic linear systems with multiplicative state noise. IEEE Transactions on Automatic Control, 42(8): 1106-1126, 1997.

[11] H. Dong, Z. Wang, and H. Gao. Distributed $\mathcal{H}_{\infty}$ filtering for a class of Markovian jump nonlinear time-delay systems over lossy sensor networks. IEEE Transactions on Industrial Electronics, 60(10): 4665-4672, 2013.

[12] H. Gao, and T. Chen. $\mathcal{H}_{\infty}$ estimation for uncertain systems with limited communication capacity. IEEE Transactions on Automatic Control, 52(11): 2070-2084, 2007.

[13] E. Gershon, U. Shaked, and I. Yaesh. Control and Estimation of State-multiplicative Linear Systems. SpringerVerlag London Limited, London, U.K., 2005.

[14] A. Germani, C. Manes, and P. Palumbo. Polynomial extended Kalman filtering for discrete-time nonlinear stochastic systems. In Proceedings of the 42nd IEEE Conference on Decision and Control, Maui, Hawaii USA, December, 886-891, 2003.

[15] A. Germani, C. Manes, and P. Palumbo. Polynomial extended Kalman filter. IEEE Transactions on Automatic Control, 50(12): 2059-2064, 2005.

[16] A. Germani, C. Manes, and P. Palumbo. Filtering of stochastic nonlinear differential systems via a Carleman approximation approach. IEEE Transactions on Automatic Control, 52(11): 2166-2172, 2007.

[17] A. Germani, C. Manes, and P. Palumbo. State estimation of stochastic systems with switching measurements: A polynomial approach. International Journal of Robust and Nonlinear Control, 19(14): 1632-1655, 2009.

[18] X. He, Z. Wang, Y. Liu, and D. H. Zhou. Least-squares fault detection and diagnosis for networked sensing systems using a direct state estimation approach. IEEE Transactions on Industrial Informatics, 9(3): 1670-1679, 2013.

[19] A. Hermoso-Carazo, and J. Linares-Pérez. Extended and unscented filtering algorithms using one-step randomly delayed observations. Applied Mathematics and Computation, 190(2): 1375-1393, 2007.

[20] J. Hu, Z. Wang, H. Gao, and L. K. Stergioulas. Extended Kalman filtering with stochastic nonlinearities and multiple missing measurements. Automatica, 48(9): 2007-2015, 2012.

[21] J. Hu, Z. Wang, B. Shen, and H. Gao. Gain-constrained recursive filtering with stochastic nonlinearities and probabilistic sensor delays. IEEE Transactions on Signal Processing, 61(5): 1230-1238, 2013.

[22] M. R. James, and I. R. Peterson. Nonlinear state estimation for uncertain systems with an integral constraint. IEEE Transactions on Signal Processing, 46,(11): 2926-2937, 1998.

[23] H. R. Karimi. Robust $\mathcal{H}_{\infty}$ filter design for uncertain linear systems over network with network-induced delays and output quantization. Modeling, Identification and Control, 30(1): 27-37, 2009.

[24] A. Q. Khan, and S. X. Ding. Threshold computation for fault detection in a class of discrete-time nonlinear systems. International Journal of Adaptive Control and Signal Processing, 25(5): 407-429, 2011. 
[25] S. Kluge, K. Reif, and M. Brokate. Stochastic stability of the extended Kalman filter with intermittent observations. IEEE Transactions on Automatic Control, 55(2): 514-518, 2010.

[26] K. Kowalski, and W. H. Steeb. Nonlinear Dynamical Systems and Carleman Linearization. Singapore: World Scientific, 1991.

[27] P. Li, J. Lam, and G. Chesi. On the synthesis of linear $\mathcal{H}_{\infty}$ filters for polynomial systems. System and Control Letters, 61(1): 31-36, 2012.

[28] G. Mavelli, and P. Palumbo. The Carleman approximation approach to solve a stochastic nonlinear control problem. IEEE Transactions on Automatic Control, 55(4): 976-982, 2010.

[29] J. Meseguer, V. Puig, T. Escobet, and J. Saludes. Observer gain effect in linear interval observer-based fault detection. Journal of Process Control, 20(8): 944-956, 2010.

[30] S. Montes de Oca, V. Puig, and J. Blesa. Robust fault detection based on adaptive threshold generation using interval LPV observers. International Journal of Adaptive Control and Signal Processing, 26(3): 258-283, 2012.

[31] V. Puig, J. Quevedo, T. Escobet, F. Nejjari, and S. de las Heras. Passive robust fault detection of dynamic processes using interval models. IEEE Transactions on Control Systems Technology, 16(5): 1083-1089, 2008.

[32] K. Reif, S. Gunther, E. Yaz, and R. Unbehauen. Stochastic stability of the discrete-time extended Kalman filter. IEEE Transactions on Automatic Control, 44(4): 714-728, 1999.

[33] B. Shen, Z. Wang, and S. X. Ding. Finite-horizon $\mathcal{H}_{\infty}$ fault estimation for linear discrete time-varying systems with delayed measurements. Automatica, 49(1): 293-296, 2013.

[34] Z. Shi, F. Gu, B. Lennox, and A. D. Ball. The development of an adaptive threshold for model-based fault detection of a nonlinear electro-hydraulic system. Control Engineering Practice, 13(17): 1357-1367, 2005.

[35] Z. Shu, J. Lam, and J. Xiong. Non-fragile exponential stability assignment of discrete-time linear systems with missing data in actuators. IEEE Transactions on Automatic Control, 54(3): 625-630, 2009.

[36] G. Wei, Z. Wang, and H. Shu. Robust filtering with stochastic nonlinearities and multiple missing measurements. Automatica, 45(3): 836-841, 2009.

[37] L. Xie, Y. C. Soh, and C. E. de Souza. Robust Kalman filtering for uncertain discrete-time systems. IEEE Transactions on Automatic Control, 39(6): 1310-1314, 1994.

[38] K. Xiong, L. Liu, and Y. Liu. Robust extended Kalman filtering for nonlinear systems with multiplicative noises. Optimal Control Applications and Methods, 32(1): 47-63, 2011.

[39] K. Xiong, C. Wei, and L. Liu. Robust extended Kalman filtering for nonlinear systems with stochastic uncertainties. IEEE Transactions on Systems, Man and Cybernetics, Part A: Systems and Humans, 40(2): 399-405, 2010.

[40] X. Zhang. Sensor bias fault detection and isolation in a class of nonlinear uncertain systems using adaptive estimation. IEEE Transactions on Automatic Control, 56(5): 1220-1226, 2011.

[41] K. Zhang, B. Jiang, and P. Shi. Fault estimation observer design for discrete-time Takagi-Sugeno fuzzy systems based on piecewise Lyapunov functions. IEEE Transactions on Fuzzy Systems, 20(1): 192-200, 2012.

[42] X. Zhang, M. Polycarpou, and T. Parisini. Robust fault isolation for a class of non-linear input-output systems. International Journal of Control, 74(13): 1295-1310, 2001.
[43] M. Zhong, S. X. Ding, J. Lam, and H. Wang. An LMI approach to design robust fault detection filter for uncertain LTI systems. Automatica, 39(3): 543-550, 2003.

[44] D. H. Zhou, X. He, Z. Wang, G. -P. Liu, and Y. D. Ji. Leakage fault diagnosis for an Internet-based three-tank system: An experimental study. IEEE Transactions on Control Systems Technology, 20(4): 857-870, 2012. 